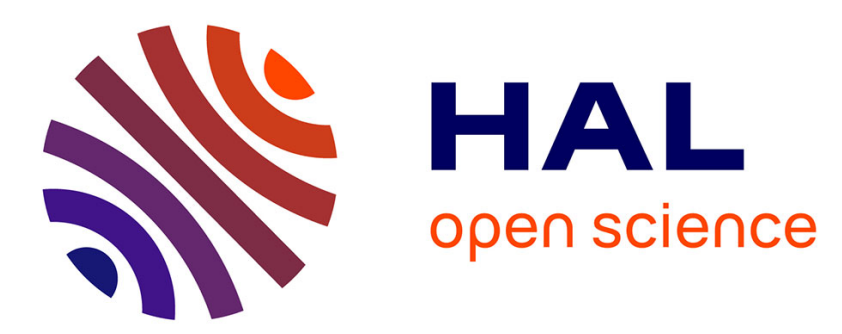

\title{
Méthodologies d'étude expérimentale et de conception assistée par ordinateur des amplificateurs de puissance à TEC GaAs (+)
}

\author{
J. Kamdem, R. Maimouni, C. Azizi, Jacques Graffeuil, P. Rossel
}

\section{> To cite this version:}

J. Kamdem, R. Maimouni, C. Azizi, Jacques Graffeuil, P. Rossel. Méthodologies d'étude expérimentale et de conception assistée par ordinateur des amplificateurs de puissance à TEC GaAs (+). Revue de Physique Appliquée, 1983, 18 (4), pp.213-227. 10.1051/rphysap:01983001804021300 . jpa-00245086

\section{HAL Id: jpa-00245086 https://hal.science/jpa-00245086}

Submitted on 1 Jan 1983

HAL is a multi-disciplinary open access archive for the deposit and dissemination of scientific research documents, whether they are published or not. The documents may come from teaching and research institutions in France or abroad, or from public or private research centers.
L'archive ouverte pluridisciplinaire HAL, est destinée au dépôt et à la diffusion de documents scientifiques de niveau recherche, publiés ou non, émanant des établissements d'enseignement et de recherche français ou étrangers, des laboratoires publics ou privés. 


\title{
Méthodologies d'étude expérimentale et de conception assistée par ordinateur des amplificateurs de puissance à TEC GaAs $\left({ }^{+}\right)$
}

\author{
J. Kamdem(*), R. Maimouni(**), C. Azizi $(* * *)$, J. Graffeuil(**) et P. Rossel(**) \\ (*) C.N.E.T.-Lannion, route de Trégastel, B.P. 40, 22301 Lannion Cedex, France \\ $\left({ }^{* *}\right)$ L.A.A.S.-C.N.R.S., 7, av. du Colonel Roche, 31400 Toulouse, France \\ (**) Université de Constantine, Algérie
}

(Reçu le 29 novembre 1982, révisé et accepté le 24 janvier 1983)

\begin{abstract}
Résumé. - Deux méthodologies d'étude des amplificateurs hyperfréquences de puissance, classe A, à transistor à effet de champ (TEC) au GaAs sont développées. On décrit, tout d'abord, une méthode expérimentale de caractérisation des TEC pour le régime des forts signaux, basée sur la notion de définition d'impédances grands signaux. On développe, en second lieu, une procédure de simulation sur ordinateur des performances de ce dispositif en régime des grands signaux à partir du modèle non linéaire du TEC GaAs élaboré au L.A.A.S. Le principe de la méthode est basé sur l'analyse dans le domaine temporel des formes d'ondes de puissances qui transitent dans le transistor. On en déduit d'une part, les courbes d'évolution de la puissance fournie à la charge en fonction de celle qui arrive sur la grille du TEC, d'autre part, les diagrammes d'impédance à gain constant, et enfin les valeurs théoriques des impédances grands signaux dont la connaissance permet de calculer la configuration des circuits d'adaptation de l'amplificateur associé. On procède, ensuite, à une comparaison théorie-expérience des résultats.
\end{abstract}

\begin{abstract}
This paper is devoted to the characterisation and to the study of the GaAs-FET properties when this device is used as a microwave power amplifier. First, an experimental method based on the large signal impedance concept, is used for determining the major parameters (input and output impedances, power gain, impedance matching levels, output power) of this device. Second, a theoretical computer-aided-design procedures is proposed : it's based on a time-domain circuit analysis for obtaining the current, voltage, and microwave power values at the device terminals. This analysis provides i) the output power versus the input power characteristics, ii) the large signal impedance contours on the Smith chart, and iii) the matching input and output circuit configuration for obtaining the best power gain or the maximum output power values. The comparison between the theoretical analysis and the experimentally obtained results is finally proposed.
\end{abstract}

1. Introduction - Le transistor à effet de champ à l'arséniure de gallium, déjà très performant dans de nombreuses applications microondes, est particulièrement sollicité depuis quelques années pour l'amplification hyperfréquence forte puissance.

La théorie classique des quadripôles linéaires et les techniques de mesure des paramètres $S$ (jusqu'à $18 \mathrm{GHz}$ ) permettent, actuellement, de prévoir le comportement du TEC GaAs en régime petits signaux et de définir les méthodes d'utilisation de ce composant dans les amplificateurs faible puissance.

Cependant, l'étude et la caractérisation de ce dispositif en régime des moyennes et fortes puissances restent difficiles et délicates, à cause :

$\left({ }^{+}\right)$Ce travail a été effectué dans le cadre du Groupement d'Etudes Coordonnées (G.R.E.C.O.) Microondes du C.N.R.S. i) de son comportement non linéaire qui est fonction de ses propriétés physiques;

ii) de la dépendance étroite de ses performances en gain et en puissance vis-à-vis du niveau des signaux et des valeurs des impédances associées aux circuits extérieurs.

Sur le plan théorique, les travaux reportés dans la littérature ont surtout porté sur une modélisation du composant en régime non linéaire $[1,2]$ associée à des applications spécifiques [3]. Sur le plan expérimental. les méthodes consistant à tracer les contours de l'impédance de charge $[4,20]$ ou à mesurer des paramètres $S$ à fort signal $[16,17]$ ont parfois été utilisées. Néanmoins, cela ne permet pas de prévoir les performances du circuit amplificateur complet.

L'absence d'une méthode rigoureuse de caractérisation ou d'identification du TEC en régime grands signaux fait que la technique de conception la plus 
usitée reste l'utilisation des paramètres $S$ petits signaux, accompagnée d'une retouche des circuits d'adaptation sur la maquette pour obtenir les performances voulues.

L'objet de cet article est de décrire, dans un premier volet, une méthode de caractérisation expérimentale et d'identification du TEC GaAs en tant qu'élément amplificateur hyperfréquence grands signaux. Cette méthode est basée sur la définition et le procédé de mesure des "impédances grands signaux " du transistor. Dans un second volet, on décrit une méthodologie de simulations sur ordinateur des amplificateurs " Classe A » de puissance à TEC, par utilisation d'un modèle mathématique non linéaire de ce dispositif. L'analyse théorique proposée est effectuée dans le domaine temporel et permet de traiter les signaux périodiques de forme complexe dans le temps. Après avoir déterminé les diagrammes théoriques d'évolution de la puissance de sortie en fonction des parties reelle et imaginaire de l'admittance de charge, on en déduit : i) les valeurs théoriques des impédances grands signaux, ii) les courbes d'impédance à gain constant, iii) les caractéristiques décrivant la puissance de sortie en fonction de celle qui entre dans le TEC. L'ensemble des résultats sera présenté sous une forme directement comparable avec les données de l'expérience.

2. Caractérisation du TEC GaAs en régime non linéaire par une méthode des impédances grands signaux. - 2.1 LES RÉGIMES DU FONCTIONNEMENT EN PUISSANCE DU TEC. - Le transistor est polarisé en régime continu par des sources de tension drainsource $V_{\mathrm{D}}$ et grille-source $V_{\mathrm{G}}$, découplées des signaux alternatifs. En absence d'excitation dynamique, le composant est parcouru par un courant de drain $I_{\mathrm{D}}$. En régime dit de classe $\mathrm{A}$, ce courant est choisi à la valeur $I_{\text {Dss }} / 2 ; I_{\text {DSs }}$ étant la valeur du courant drain à saturation obtenu pour la tension grille-source nulle.

En régime dynamique, pour une fréquence donnée et pour des impédances de source et de charge fixées, la caractéristique qui représente la puissance de sortie $p_{\mathrm{s}}$ en fonction de la puissance $p_{\mathrm{e}}$ qui entre dans le TEC, présente en coordonnées logarithmiques $(\mathrm{dBm})$ l'allure typique du tracé de la figure 1 . Le fonctionnement, dit linéaire, est caractérisé par une portion de droite (I), de pente unité, dont l'ordonnée à l'origine, fournit le gain petit signal en $\mathrm{dB}$. La portion (II) incurvée traduit le comportement non linéaire du dispositif, de sorte que l'écart entre chacun de ces points et le prolongement de la portion de droite exprime en $\mathrm{dB}$ l'état de compression en puissance du transistor. Cet effet de saturation est dû aux nonlinéarités du transistor qui apparaissent dès que les excursions en tension et en courant atteignent les régions courbées des caractéristiques statiques (Fig. 2), provoquant à la fois une diminution de la transconductance $\left(\Delta I_{\mathrm{D}} / \Delta V_{\mathrm{GS}}\right) V_{\mathrm{DS}}$ et une augmentation de la conductance de sortie $\left(\Delta I_{\mathrm{D}} / \Delta V_{\mathrm{DS}}\right) V_{\mathrm{GS}}$. Il en résulte une déformation des signaux et l'apparition, dans le dispositif, de signaux à fréquences harmoniques dont

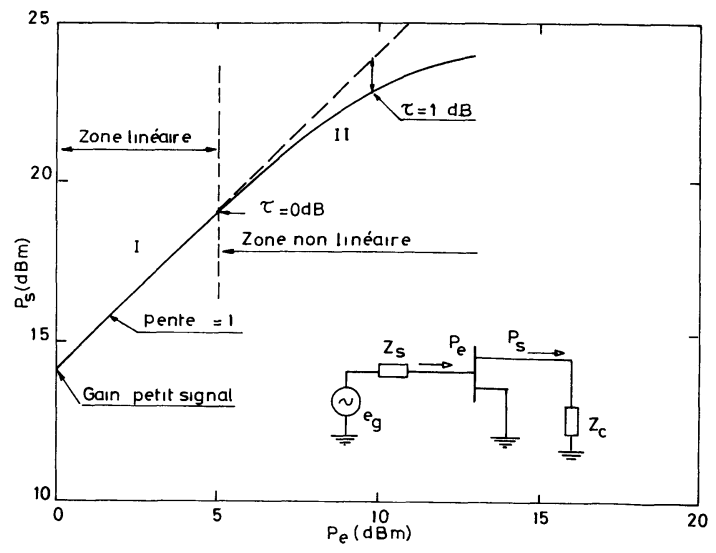

Fig. 1. - Allure typique de la caractéristique $P_{\mathrm{s}}\left(P_{\mathrm{e}}\right)$ d'un étage amplificateur de puissance hyperfréquence.

[Output power versus input power characteristic in a microwave power amplifier.]

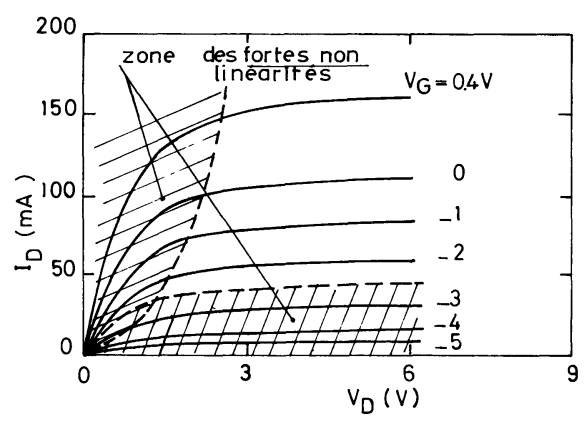

Fig. 2. - Caractéristiques courant-tension. Mise en évidence des régions génératrices des non-linéarités dans le transistor.

[Drain-current versus drain-voltage characteristics showing the non-linear regions.]

l'importance croît avec l'augmentation de la puissance incidente sur la grille [4].

2.2 DÉFINITION DES IMPÉDANCES ADAPTÉES. - Sur le schéma de la figure 3 , nous rappelons la configuration d'un étage amplificateur. Celui-ci est constitué d'un générateur de force électromotrice $e_{\mathrm{g}}$, d'impédance interne $Z_{\mathrm{s}}$, délivrant des tensions sinusoïdales de fréquence $F_{0}$, d'un composant actif le TEC GaAs qui constitue l'élément amplificateur et d'un récepteur constitué d'une charge d'impédance $Z_{\mathrm{c}}$. Le problème que l'on se pose est de transmettre, par l'intermédiaire du transistor, le maximum de puissance du générateur vers la charge. Pour cela, il faut que le générateur puisse injecter le maximum de puissance sur la grille et, d'autre part, que la charge reçoive le maximum de puissance retirable du TEC. Ceci conduit à définir les impédances que doit voir le transistor côté entrée, c'est-à-dire sur sa grille et, côté sortie, sur son drain.

Le principe de l'adaptation est d'obtenir le gain de transfert maximum en puissance. Il consiste à placer, d'une part entre le générateur et l'entrée 

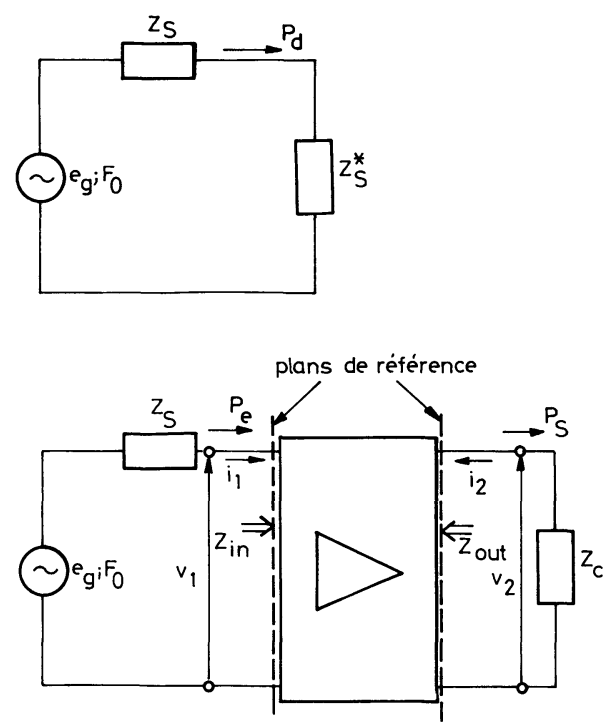

Fig. 3. - Définition des impédances et des puissances en régime grands signaux dans un quadripôle amplificateur en régime non linéaire.

[Definition of impedances and of power values for a quadripole.] du transistor, d'autre part entre la charge et la sortie du composant, des circuits non dissipatifs, tels que le transistor voie sur ses bornes des impédances qui, suivant le régime du fonctionnement en zone linéaire ou en zone de compression, sont définies de la façon suivante :

2.2.1 Impédances adaptées au transistor en régime linéaire dynamique petit signal. - Pour une fréquence d'étude donnée, si le transistor fonctionne en régime dynamique tel que l'amplitude de la f.e.m. du générateur ait une valeur suffisamment faible pour que les signaux, $\left(i_{1}, v_{1}\right)$ à l'entrée et $\left(i_{2}, v_{2}\right)$ à la sortie, soient sinusoïdaux et répondent au principe de superposition, on définit les impédances adaptées, $Z_{\mathrm{s}}$ et $Z_{\mathrm{c}}$ que voit le transistor, comme les conjuguées de ses impédances d'entrée, $Z_{\text {in }}$, ou de sortie, $Z_{\text {out }}$; sous la formulation d'admittance $\left(Y_{\mathrm{s}}=Z_{\mathrm{s}}^{-1}\right.$; $\left.Y_{\mathrm{c}}=Z_{\mathrm{c}}^{-1}\right)$. Le transfert optimal, lorsque le transistor est inconditionnellement stable, est obtenu lorsque les égalités suivantes sont satisfaites :

$$
\left.\begin{array}{l}
\mathrm{R}_{\mathrm{e}}\left(Y_{\mathrm{s}}\right)=\frac{1}{2 \mathrm{R}_{\mathrm{e}}\left(Y_{22}\right)}\left\{\left[2 \mathrm{R}_{\mathrm{e}}\left(Y_{11}\right) \cdot \mathrm{R}_{\mathrm{e}}\left(Y_{22}\right)-\mathrm{R}_{\mathrm{e}}\left(Y_{21} \cdot Y_{12}\right)\right]^{2}-\left|Y_{21} \cdot Y_{12}\right|^{2}\right\}^{1 / 2} \\
\mathrm{I}_{\mathrm{m}}\left(Y_{\mathrm{s}}\right)=-\mathrm{I}_{\mathrm{m}}\left(Y_{11}\right)+\frac{\mathrm{I}_{\mathrm{m}}\left(Y_{21} \cdot Y_{12}\right)}{2 \mathrm{R}_{\mathrm{e}}\left(Y_{22}\right)} \\
\mathrm{R}_{\mathrm{e}}\left(Y_{\mathrm{c}}\right)=\frac{1}{2 \mathrm{R}_{\mathrm{e}}\left(Y_{11}\right)}\left\{\left[2 \mathrm{R}_{\mathrm{e}}\left(Y_{11}\right) \mathrm{R}_{\mathrm{e}}\left(Y_{22}\right)-\mathrm{R}_{\mathrm{e}}\left(Y_{21} \cdot Y_{12}\right)\right]^{2}-\left|Y_{21} \cdot Y_{12}\right|^{2}\right\}^{1 / 2} \\
\mathrm{I}_{\mathrm{m}}\left(Y_{\mathrm{c}}\right)=-\mathrm{I}_{\mathrm{m}}\left(Y_{22}\right)+\frac{\mathrm{I}_{\mathrm{m}}\left(Y_{21} \cdot Y_{12}\right)}{2 \mathrm{R}_{\mathrm{e}}\left(Y_{11}\right)},
\end{array}\right\}
$$

où $R_{e}$ et $I_{m}$ désignent respectivement la partie réelle et la partie imaginaire, et $Y_{i j}$ sont les éléments de la matrice admittance du composant. Ces admittances adaptées dépendent seulement des paramétres admittances $Y_{i j}$ du transistor.

Par ailleurs, dans ce régime, les impédances à l'entrée $Z_{\text {in }}$ et à la sortie $Z_{\text {out }}$ ne posent aucune ambiguité de définition; tous les signaux sont sinusoïdaux et l'on a :

$$
\left.\begin{array}{l}
Z_{\text {in }}=\frac{v_{1}}{i_{1}} \\
Z_{\text {out }}=\frac{v_{2}}{i_{2}} .
\end{array}\right\}
$$

2.2.2 Impédances adaptées au transistor en régime dynamique grand signal. - Lorsque la f.e.m. du générateur $e_{\mathrm{g}}$ reste sinusoïdale et est telle que les signaux à l'entrée $\left(i_{1}, v_{1}\right)$ et à la sortie $\left(i_{2}, v_{2}\right)$ du transistor sont périodiques, mais deviennent de forme non sinusoïdale, on définit les impédances grands signaux du transistor. Cette notion d'impédances grands signaux dérive de la définition d'impédances communément admise en régime périodique temporel sinusoïdal et permet de caractériser un étage amplificateur évoluant en régime non linéaire. Ces impédances qui ne sont qu'un abus de langage, appelées $Z_{\text {in }}$ et $Z_{\text {out }}$, sont définies comme étant les conjuguées des valeurs particulières des impédances du générateur, $Z_{\mathrm{s}}$, et de la charge, $Z_{\mathrm{c}}$, prises lorsque se produit une dissipation maximale de la puissance dans $Z_{c}$, et ceci pour une valeur particulière de la puissance retirable du générateur $\left(e_{\mathrm{g}}-Z_{\mathrm{s}}\right)$ :

$$
\begin{aligned}
& Z_{\text {in }}=Z_{\mathrm{s}}^{*}, \\
& Z_{\text {out }}=Z_{\mathrm{c}}^{*} .
\end{aligned}
$$

Cela implique que ces impédances soient des fonctions de la valeur de la puissance retirable du générateur ou de la puissance fournie à la charge.

De par leur définition, les impédances $Z_{\mathrm{s}}$ et $Z_{\mathrm{c}}$ doivent en toute rigueur présenter d'une part une 
certaine configuration et avoir d'autre part des valeurs telles que les diverses composantes, fondamentale (à la fréquence du générateur de source) et ses harmoniques, générés à la sortie par les non linéarités $\mathrm{du}$ système, émettent simultanément le maximum de puissance vers la charge.

2.2.3 Définition des diverses puissances. - Les diverses puissances qui transitent dans le circuit amplificateur de la figure 3 sont :

(i) La puissance maximale retirable du générateur notée $P_{\mathrm{d}}$ :

$$
P_{\mathrm{d}}=\frac{E^{2}}{4 \mathrm{R}_{\mathrm{e}}\left(Z_{\mathrm{s}}\right)},
$$

où $E$ est la valeur efficace de la tension du générateur de source $e_{\mathrm{g}}$;

(ii) La puissance qui rentre effectivement dans le transistor, notée $P_{\mathrm{e}}$, et qui est définie en régime permanent par la formule :

$$
P_{\mathrm{e}}=\frac{1}{T} \int_{0}^{\mathrm{T}} v_{1}(t) \cdot i_{1}(t) \mathrm{d} t
$$

Elle est obtenue par intégration du signal correspondant au produit des formes d'ondes instantanées de courant et de tension $\left(v_{1}, i_{1}\right)$ sur une période $T$ de ce signal;

(iii) La puissance de sortie, notée $P_{\mathrm{s}}$, qui représente la puissance fournie par le transistor et dissipée dans la charge. Elle est définie de manière analogue à la puissance $P_{\mathrm{e}}$ :

$$
P_{\mathrm{s}}=\frac{1}{T} \int_{0}^{\mathrm{T}} v_{2}(t) \cdot i_{2}(t) \mathrm{d} t
$$

$v_{2}(t)$ et $i_{2}(t)$ sont respectivement la tension et le courant instantanés dans l'impédance de charge $Z_{\mathrm{c}}$ et $T$ la période du signal $\left(v_{2}(t) . i_{2}(t)\right)$.

2.3 LA MÉTHODE DE CARACTÉRISATION EXPÉRIMENTALE. - Le principe de la méthode consiste, pour chaque fréquence et pour des conditions de polarisations continues fixées, à faire varier la puissance $P_{\mathrm{d}}$ retirable du générateur d'entrée et à déterminer les valeurs des impédances grands signaux du transistor $Z_{\text {in }}$ et $Z_{\text {out }}$, ainsi que les valeurs de la puissance de sortie $P_{\mathrm{s}}$ qui leur est associée, compte tenu de la définition du paragraphe 2.2.
Le dispositif expérimental est un banc hyperfréquence (Fig. 4) d'impédance caractéristique égale à $50 \Omega$; il constitue, avec le transistor sous test, un amplificateur de puissance dont les impédances de source $Z_{\mathrm{s}}$ et de charge $Z_{\mathrm{c}}$ sont manuellement réglables à l'aide de deux réacteurs hyperfréquences non dissipatifs. La source de puissance est capable de fournir des niveaux de puissance, permettant de soumettre le transistor à des taux de compression allant de 0

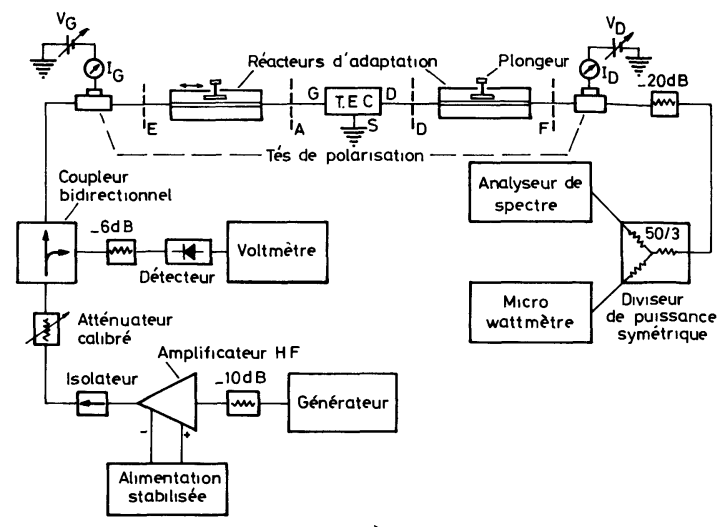

a)

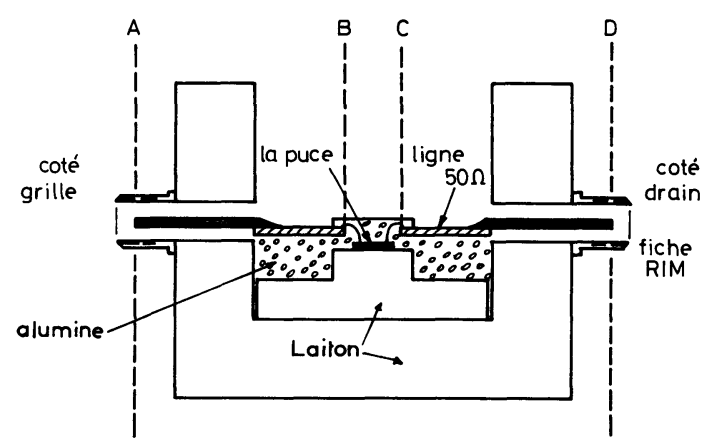

b)

Fig. 4. - (a) Banc expérimental de mesures hyperfréquences. (b) Monture des transistors.

[The experimental measurement configuration and the device fixture.]

à $6 \mathrm{~dB}$. L'étude a été menée sur deux types de transistors : GAT $1(Z=360 \mu \mathrm{m})$ de faible puissance, et THC $302(Z=800 \mu \mathrm{m})$ de moyenne puissance. Leurs paramètres physiques : dopage, mobilité, longueur et largeur de canal, épaisseur de couche conductrice, facteur de pente, tension de seuil et résistance thermique, sont rassemblés dans le tableau I.

Tableau I. - Valeurs des paramètres physiques et géométriques des transistors.

[Physical and geometrical parameters of the devices.]

\begin{tabular}{|l|c|c|c|c|c|c|c|c|}
\hline & $\begin{array}{c}N_{\mathrm{D}} \\
\left(\mathrm{cm}^{-3}\right)\end{array}$ & $\begin{array}{c}\mu_{0} \\
\left(\mathrm{~cm}^{2} / \mathrm{Vs}\right)\end{array}$ & $\begin{array}{c}L \\
(\mu \mathrm{m})\end{array}$ & $\begin{array}{c}Z \\
(\mu \mathrm{m})\end{array}$ & $\begin{array}{c}d \\
(\mu \mathrm{m})\end{array}$ & $\begin{array}{c}G_{0} \\
(\mathrm{mV})\end{array}$ & $\begin{array}{c}V_{\mathrm{T}} \\
(\mathrm{V})\end{array}$ & $\begin{array}{c}R_{\mathrm{th}} \\
(\% / \mathrm{W})\end{array}$ \\
\hline THC 302 (98) & $2 \times 10^{17}$ & 1700 & 1 & 800 & 0,2 & 780 & $-4,5$ & 130 \\
\hline GAT 1 432 LID & $6 \times 10^{16}$ & 3800 & 4 & 360 & 0,3 & 90 & $-3,3$ & 980 \\
\hline
\end{tabular}


2.3.1 Les caractéristiques en puissance $P_{\mathrm{s}}\left(\boldsymbol{P}_{\mathrm{d}}\right)$. Ces courbes décrivent, pour des conditions de polarisation figées, l'évolution de la puissance de sortie $P_{\mathrm{s}}$ en fonction de celle, $P_{\mathrm{d}}$, qui est disponible sur le générateur d'entrée; elles sont paramétrées par les valeurs du couple $\left(Z_{\mathrm{s}}, Z_{\mathrm{c}}\right)$ d'impédances de source et de charge.

Sur les figures 5 et 6 représentant un exemple des courbes obtenues à 3 et $6 \mathrm{GHz}$, deux types de caractéristiques en puissances sont tracés :

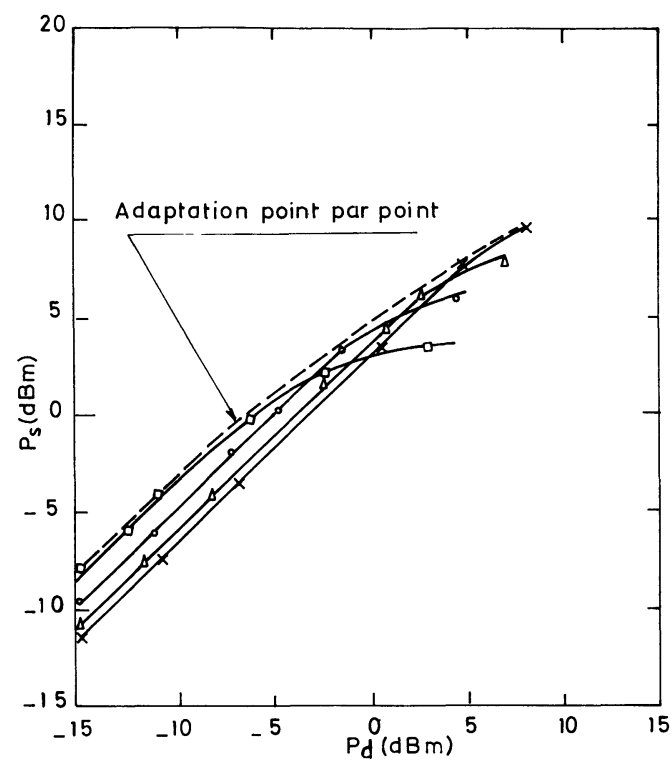

Fig. 5. - La caractéristique en puissance $P_{s}\left(P_{d}\right)$, avec adaptation point par point, est une enveloppe de celles avec adaptation en un seul point. $\Delta$ : adaptation au point d'abscisse $P_{\mathrm{d}}=5 \mathrm{dBm}, 0$ : adaptation au point d'abscisse $P_{\mathrm{d}}=2 \mathrm{dBm}, \diamond$ : adaptation au point d'abscisse $\boldsymbol{P}_{\mathrm{d}}=$ $-10 \mathrm{dBm}, \times$ : adaptation au point d'abscisse $P_{\mathrm{d}}=$ 6,5 dBm, GAT 1 (432), $F_{0}=3 \mathrm{GHz}, V_{\mathrm{D}}=4 \mathrm{~V}, I_{\mathrm{D}}=$ $\mathrm{I}_{\mathrm{DSs}} / 2=15 \mathrm{~mA}$.

$\left[P_{\mathrm{s}} \quad\right.$ vs. $P_{\mathrm{e}}$ characteristics obtained for various matching conditions at the output terminals.]

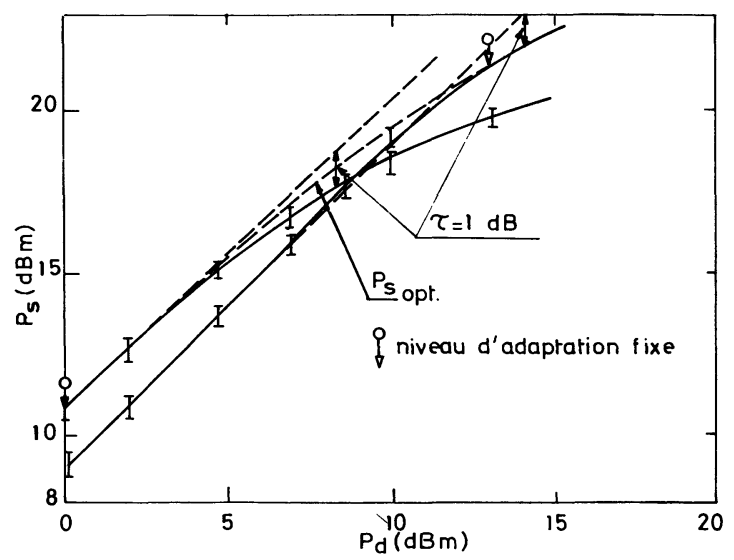

Fig. 6. - Caractéristiques puissance de sortie en fonction de la puissance disponible au générateur d'entrée. THC $302(18), F_{0}=6 \mathrm{GHz}, V_{\mathrm{G}}=-2 \mathrm{~V}, I_{\mathrm{D}}=\mathrm{I}_{\mathrm{DSs}} / 2$, $V_{\mathrm{D}}=5 \mathrm{~V}$.

[Output-power characteristics as a function of the power available from the input-generator.] i) Pour les courbes en trait plein, l'adaptation de $Z_{\mathrm{s}}$ et $Z_{\mathrm{c}}$ à l'aide de réacteurs hyperfréquences pour un transfert maximum de puissance vers ta charge est réalisée pour un seul point de chaque courbe et n'est plus modifiée pour tout le tracé. Pour chaque fréquence, le transistor est ainsi caractérisé par une famille de courbes en puissance, $P_{\mathrm{s}}\left(\boldsymbol{P}_{\mathrm{d}}\right)$, dont le paramètre est la valeur du couple d'impédances $\left(Z_{s}, Z_{c}\right)$ de source et de charge correspondant au point où le transfert optimal de puissance vers la charge a été choisi. Par définition, ce couple d'impédances est, en ce point, choisi égal au complexe conjugué du couple d'impédances grands signaux du transistor qui ont été notées $Z_{\text {in }}$ et $Z_{\text {our }}$. Nous décrivons, dans le paragraphe suivant, la méthode de détermination expérimentale de $Z_{\text {in }}$ et $Z_{\text {our }}$ On note que sur ces courbes (Figs. 5 et 6), la zone de fonctionnement en régime linéaire est d'autant plus étendue que la valeur de la puissance disponible, $\boldsymbol{P}_{\mathrm{d}}$, pour laquelle ce point optimum est pris, est élevée. Corrélativement, on observe que plus le choix de ce point est fait à puissance élevée, plus le gain en puissance petits signaux diminue.

ii) Les courbes en pointillé sont obtenues en adaptant, pour chaque valeur de $\boldsymbol{P}_{\mathrm{d}}$, le couple d'impédances $\left(Z_{\mathrm{s}}, Z_{\mathrm{c}}\right)$ à un transfert optimal de puissance vers la charge. Ces courbes, avec adaptation point par point, sont les enveloppes de celles qui correspondent à l'adaptation en un seul point décrites précédemment. Par ailleurs, contrairement au cas précédent, le transistor ne possède, pour un point de repos continu fixé et à fréquence imposée, qu'une seule caractéristique en puissance avec adaptation point par point. Cette caractéristique est la seule qui traduise les possibilités optimales en valeurs de puissance retirable du transistor; sa zone de saturation est due aux effets non linéaires internes au composant.

Une caractéristique de puissance, avec adaptation point par point, est donc l'élément de choix pour la comparaison de plusieurs transistors de puissance.

2.3.2 Les impédances grands signaux, méthode de mesure et propriétés. - Le dispositif de mesure est le banc hyperfréquence de la figure 4 associé à un analyseur de réseau pour la mesure des impédances linéaires.

Pour une fréquence $F_{0}$ de la source, le TEC est soumis à un niveau de puissance disponible $P_{\mathrm{d}}$. Les systèmes d'adaptation d'impédances (Fig. 4) sont alors réglés pour un transfert optimal de puissance à travers le banc [5]. Dans une étape suivante, les systèmes d'adaptation dont les configurations géométriques sont conservées, sont retirés du banc hyperfréquence, puis chargés aux accès $E$ et $F$ (Fig. 4) par une charge de $50 \Omega$. Leurs coefficients de réflexion sont ensuite mesurés sur un pont de paramètres $S$ à la fréquence $F_{0}$ dans les plans $\mathrm{A}$ et $\mathrm{D}$ (Fig. 4). Le report de ces coefficients de réflexion sur l'abaque 
de Smith fournit les impédances vues par la puce du transistor dans les plans A et D (Fig. 7). Par transformation d'impédance, on ramène les impédances précédentes dans les plans $\mathrm{B}$ et $\mathrm{C}$ au ras de la puce.

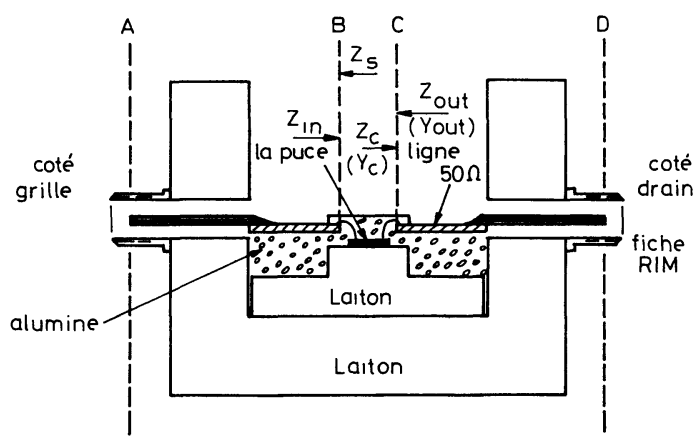

Fig. 7. - Monture des transistors. Mise en évidence des plans de référence et diverses impédances et admittances.

[The transistor fixture where the reference planes and the various impedances are indicated.]

On obtient ainsi les impédances de source $Z_{\mathrm{s}}$ et de charge $Z_{\mathrm{c}}$, dont les conjuguées complexes sont égales aux impédances grands signaux du TEC, $Z_{\text {in }}$ et $Z_{\text {out }}$. Dans le plan complexe, nous les noterons :

$$
\begin{aligned}
& Z_{\text {in }}=\mathrm{R}_{\mathrm{e}}\left(Z_{\text {in }}\right)+j \mathrm{I}_{\mathrm{m}}\left(Z_{\text {in }}\right), \\
& Z_{\text {out }}=\mathrm{R}_{\mathrm{e}}\left(Z_{\text {out }}\right)+j \mathrm{I}_{\mathrm{m}}\left(Z_{\text {out }}\right),
\end{aligned}
$$

où $j$ est le symbole de l'imaginaire pur. Les admittances grands signaux correspondantes sont définies par :

$$
\begin{aligned}
& Y_{\text {in }}=\frac{1}{Z_{\text {in }}}, \\
& Y_{\text {out }}=\frac{1}{Z_{\text {out }}} .
\end{aligned}
$$

Un exemple des résultats obtenus sur le transistor THC 302 (98) à $4 \mathrm{GHz}$ est reporté sur la figure 8 pour la puissance disponible du générateur d'entrée variant de 1 à $20 \mathrm{~mW}$. Sur l'ensemble des résultats obtenus à diverses fréquences [5], nous avons pu observer trois propriétés spécifiques des impédances grands signaux lorsqu'on fait croître la puissance disponible sur l'entrée : i) L'impédance grand signal de sortie $Z_{\text {out }}$ varie relativement beaucoup plus que celle d'entrée $Z_{\text {in }}$. Dans le cas de la figure 8 , on note que $\mathrm{R}_{\mathrm{e}}\left(Z_{\text {in }}\right)$ reste constant, tandis que $\mathrm{R}_{\mathrm{e}}\left(Z_{\text {out }}\right)$ augmente de $60 \%$. De même $\mathrm{I}_{\mathrm{m}}\left(Z_{\text {in }}\right)$ diminue de $30 \%$, alors que $\mathrm{I}_{\mathrm{m}}\left(Z_{\text {out }}\right)$ est multiplié par un facteur 5 . ii) La partie imaginaire de l'admittance de sortie grand signal $Y_{\text {out }}$ reste sensiblement constante autour de la valeur qui est la sienne en régime petits signaux. iii) Pour les valeurs suffisamment élevées de la puissance $\boldsymbol{P}_{\mathrm{d}}$, la partie réelle de l'admittance de sortie grand signal tend vers une valeur constante.

Ainsi, en tant qu'application des méthodes expéri-

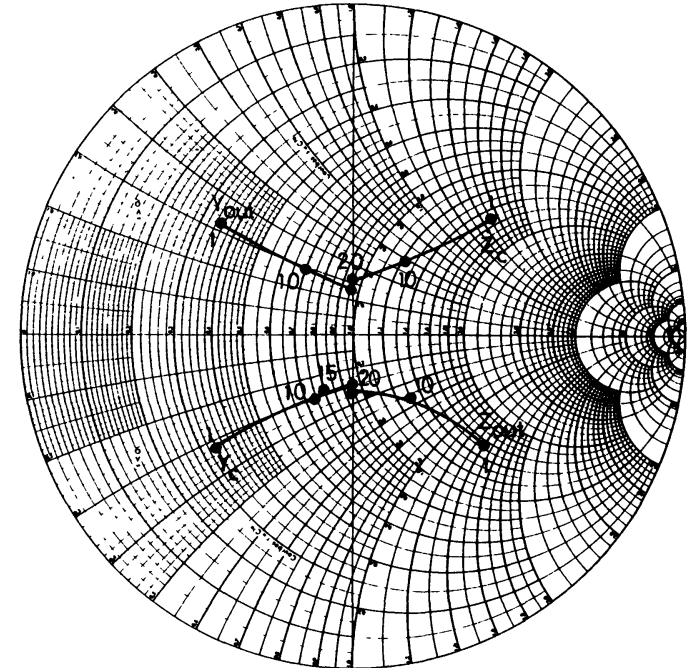

a)

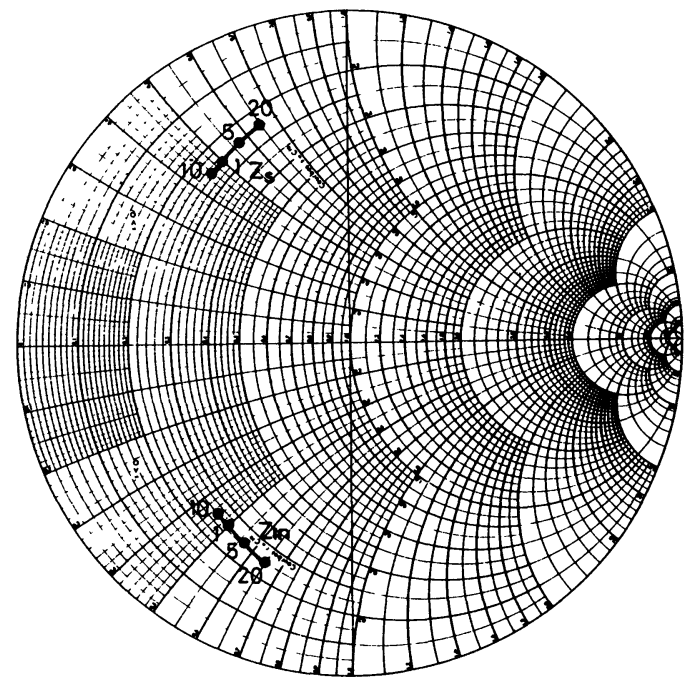

b)

Fig. 8. - Variations expérimentales des impédances et admittances grands signaux en fonction du niveau de la puissance disponible $P_{\mathrm{d}}$, THC $302(98), V_{\mathrm{D}}=7 \mathrm{~V}, I_{\mathrm{D}}=$ $I_{\mathrm{DSS}} / 2, V_{\mathrm{G}}=-2 \mathrm{~V}, F_{0}=4 \mathrm{GHz}$, le paramètre est $P_{\mathrm{d}}$.

[Experimental values of the large signal impedances or admittances as a function of the available power $P_{\mathrm{d}}$.]

mentales de caractérisation du TEC qui viennent d'être développées ici, il est possible de déterminer toutes les principales grandeurs qui sont à la base de la conception des amplificateurs hyperfréquences de puissance à TEC GaAs : i) La puissance maximale que peut fournir le transistor, compte tenu du taux de compression admissible, ainsi que ii) Le gain en puissance en régime linéaire ou non linéaire, sont fournis par les caractéristiques puissance de sortie-puissance disponible (Figs. 5 et 6), avec adaptation point par point ou avec adaptation en un seul point, iii) La connaissance des impédances grands signaux permet de calculer la configuration des 
circuits d'adaptation qui doivent être placés à l'entrée et à la sortie du transistor, compte tenu des performances recherchées.

3. Méthode de simulation en grands signaux des amplificateurs hyperfréquences à TEC GaAs. Comparaison théorie-expérience. - Dans cette partie, nous utilisons le modèle non linéaire du TEC GaAs qui a été développé antérieurement au L.A.A.S. [6, 7], pour rendre compte des propriétés en grands signaux du composant en tant qu'élément amplificateur de puissance. A partir de la méthodologie de simulation proposée, nous nous attacherons à retrouver sur le plan théorique, les propriétés des impédances grands signaux, les caractéristiques en puissance et les diagrammes d'impédances de charge à gain constant. L'analyse sera effectuée dans le domaine temporel où les signaux sont exploités dans leurs formes périodiques complexes.

3.1 LE MODÈLE GRAND SIGNAL DU TEC GaAs. Ce modèle qui a été décrit dans d'autres publications est du type non linéaire. Il est basé sur l'analyse des principaux mécanismes physiques internes qui régissent le fonctionnement du dispositif [8-10] et les relations mathématiques permettant de les décrire [7, 10, 11]. Il conduit au schéma électrique décrit sur la figure 9 et prend en compte, d'une part le transistor

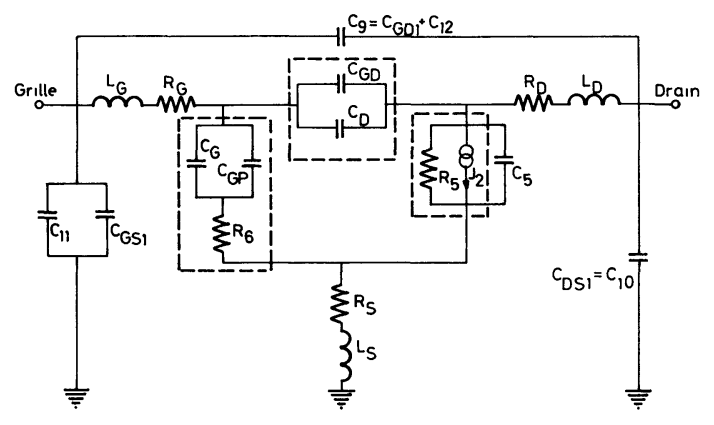

Fig. 9. - Schéma équivalent du modèle grand signal. Les éléments non linéaires sont entourés de pointillés et représentent le transistor intrinsèque.

[Equivalent circuit of the GaAs FET. The non-linear elements are included in the dotted contour.]

intrinsèque synthétisé par les éléments non linéaires (entourés de pointillés) et, d'autre part les éléments dits parasites liés principalement aux contraintes de montage sur embase. Les expressions mathématiques décrivant ces éléments non linéaires [7, 13, 15], que nous ne rappellerons pas ici, sont des fonctions explicites des tensions internes grille-source et grille-drain et ont été établies en prenant en compte le compromis entre la complexité et la précision, ce qui rend ce modèle compatible en formulation et en topologie avec les logiciels d'analyse des circuits analogiques, sur ordinateur.
La validité de ce modèle a été vérifiée sur divers types de transistors, par simulation dans le programme IMAG III [18], i) en régime statique, par comparaison des caractéristiques courant-tension théoriques et expérimentales $[6,7]$, ii) en régime dynamique linéaire sinusoïdal, par la mesure et le calcul des paramètres d'onde $S_{i j}$ et des paramètres admittance $Y_{i j}$ [11], iii) en régime dynamique non linéaire, en comparant les composantes harmoniques de puissance générées par le transistor lorsqu'il est monté en amplificateur simple étage constitué d'un générateur d'impédance $50 \Omega$ et d'une charge $50 \Omega$, selon la technique introduite en 1978 par H. A. Willing [12]. Cette procédure est la mieux adaptée à la vérification d'un modèle de composant en régime non linéaire. La figure 10 est un exemple des résultats obtenus par cette méthode de caractérisation [4].

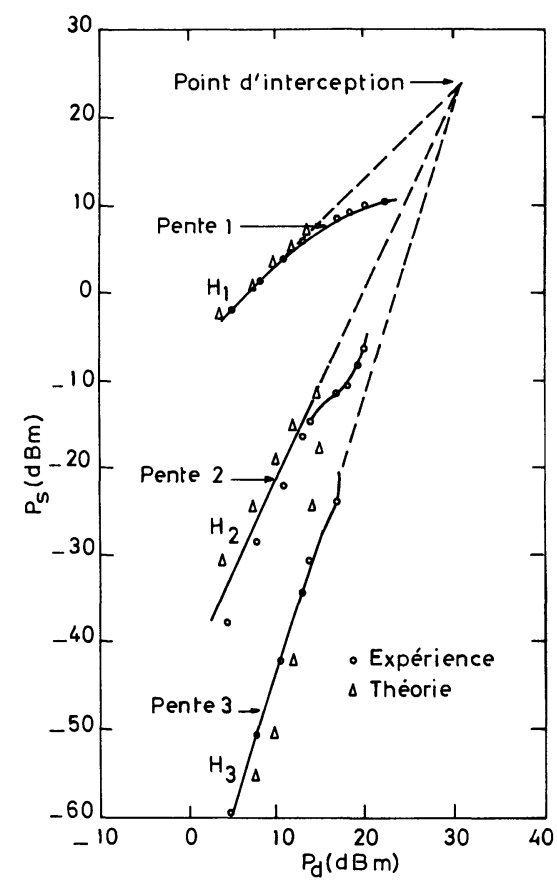

Fig. 10. - Composantes harmoniques de la puissance de sortie en fonction de la puissance disponible à l'entrée dans un système $50 \Omega$, GAT $1(432), V_{\mathrm{D}}=4 \mathrm{~V}, I_{\mathrm{D}}=I_{\mathrm{DSS}} / 2$, $F_{0}=1 \mathrm{GHz}$

[Harmonic decomposition of the output power as a function of the input available power.]

\subsection{IMPLANTATION D'UN ÉTAGE AMPLIFICATEUR DE} PUISSANCE DANS LE LOGICIEL IMAG III. - La procédure suivie comporte les étapes suivantes :

i) En régime petits signaux, les paramètres $Y_{i j}$ théoriques du TEC sont déterminés dans le logiciel IMAG III [18], à partir du modèle non linéaire décrit sur la figure $9 a$ pour des conditions de polarisation figées $\left(V_{\mathrm{D}}, I_{\mathrm{DSs}} / 2\right)$. Il est utilisé, dans ce cas particulier, en régime alternatif sinusoïdal [5] dans les configurations de circuits qui correspondent à la définition des paramètres $Y_{i j}$. 
ii) On calcule, ensuite, les valeurs des admittances de source $Y_{\mathrm{s}}$ et de charge $Y_{\mathrm{c}}$, pour lesquelles l'étage amplificateur schématisé sur la figure 11 se trouve adapté en impédance en régime petits signaux. Ces impédances sont fournies par la connaissance des paramètres $Y_{i j}$ du quadripôle linéaire équivalent suivant les relations :

$$
\begin{aligned}
& \mathrm{R}_{\mathrm{e}}\left(Y_{\mathrm{c}}\right)=\frac{\left|Y_{12} \cdot Y_{21}\right|}{2 \mathrm{R}_{\mathrm{e}}\left(Y_{11}\right)} \sqrt{K^{2}-1}, \\
& \mathrm{I}_{\mathrm{m}}\left(Y_{\mathrm{c}}\right)=\frac{\mathrm{I}_{\mathrm{m}}\left(Y_{12} \cdot Y_{21}\right)}{2 \mathrm{R}_{\mathrm{e}}\left(Y_{11}\right)}-\mathrm{I}_{\mathrm{m}} Y_{22},
\end{aligned}
$$

où

$$
\begin{aligned}
K= & \frac{1}{\left|Y_{12} \cdot Y_{21}\right|} \times \\
& \times\left[2 \cdot \mathrm{R}_{\mathrm{e}}\left(Y_{11}\right) \cdot \mathrm{R}_{\mathrm{e}}\left(Y_{22}\right)-\mathrm{R}_{\mathrm{e}}\left(Y_{12} \cdot Y_{21}\right)\right],
\end{aligned}
$$

où $R_{e}$ désigne la partie réelle et $I_{m}$ la partie imaginaire de la quantité entre parenthèses. $Y_{\mathrm{s}}$ s'obtient en permutant les indices 1 et 2 . Le tableau II regroupe, en tant qu'exemple, les valeurs de ces admittances dans le cas du transistor THC 302 (98) à 4 et $5 \mathrm{GHz}$;

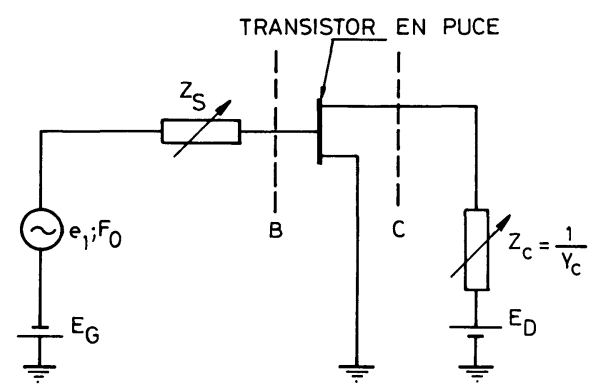

Fig. 11. - Schéma général d'un amplificateur de puissance simple étage à TEC.

[Configuration of a single stage power amplifier.]

iii) de ce calcul, on déduit par synthèse le réseau électrique représentant l'admittance de charge $Y_{\mathrm{c}}$ et l'impédance de source $Z_{\mathrm{s}}$, telle que $Z_{\mathrm{s}}=Y_{\mathrm{s}}^{-1}$, sous la forme d'éléments discrets. Pour les transistors étudiés, on obtient les configurations de réseaux

Tableau II. - Impédance de source $Z_{\mathrm{s}}$ et admittance de charge $Y_{\mathrm{c}}$ adaptées en régime de petits signaux.

THC $302(98), V_{\mathrm{D}}=7 \mathrm{~V}, I_{\mathrm{D}}=120 \mathrm{~mA}, V_{\mathrm{G}}=-2 \mathrm{~V}$.

[Input impedance and load admittance values in the small signal assumption.]

\begin{tabular}{|c|c|c|c|c|}
\hline $\begin{array}{c}F \\
(\mathrm{GHz})\end{array}$ & $\begin{array}{c}\mathrm{R}_{\mathrm{e}}\left(Z_{\mathrm{s}}\right) \\
(\Omega)\end{array}$ & $\begin{array}{c}\mathrm{I}_{\mathrm{m}}\left(Z_{\mathrm{s}}\right) \\
(\Omega)\end{array}$ & $\begin{array}{c}\mathrm{R}_{\mathrm{e}}\left(Y_{\mathrm{c}}\right) \\
(\mathrm{mV})\end{array}$ & $\begin{array}{c}\mathrm{I}_{\mathrm{m}}\left(Y_{\mathrm{c}}\right) \\
(\mathrm{mV})\end{array}$ \\
\hline 4 & 2,5 & 38 & 20 & $-21,5$ \\
\hline 5 & 10,6 & 31,4 & 8,7 & $-20,8$ \\
\hline
\end{tabular}

données sur la figure 12, dans lesquelles les résistances $R_{\mathrm{s}}$ et $R_{\mathrm{c}}$ et les inductances $L_{\mathrm{s}}$ et $L_{\mathrm{c}}$ s'écrivent :

$$
\begin{aligned}
& R_{\mathrm{s}}=\mathrm{R}_{\mathrm{e}}\left(Z_{\mathrm{s}}\right) \\
& L_{\mathrm{s}}=\frac{\mathrm{I}_{\mathrm{m}}\left(Z_{\mathrm{s}}\right)}{\omega} \\
& R_{\mathrm{c}}=\frac{1}{\mathrm{R}_{\mathrm{e}}\left(Y_{\mathrm{c}}\right)} \\
& L_{\mathrm{c}}=\frac{1}{\mathrm{I}_{\mathrm{m}}\left(Y_{\mathrm{c}}\right) \cdot \omega} .
\end{aligned}
$$

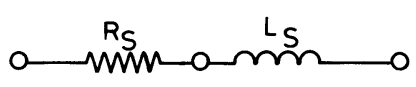

$\left[z_{s}\right]$

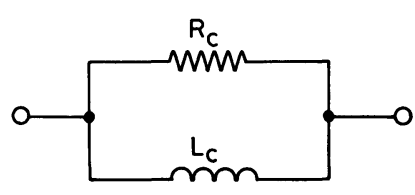

$\left[\mathrm{Y}_{\mathrm{C}}\right]$

Fig. 12. - Représentation possible de la configuration des impédances de source et de charge pour le transfert maximum de puissance THC 302, GAT 1432.

[Input and output impedances configuration used for the numerical simulation.]

Cette configuration des réseaux d'adaptation ne satisfait pas en toute rigueur au critère de définition du paragraphe 2.2. Elle est valable en régime linéaire, et pour être conservée lorsque l'étage amplificateur évolue en régime de compression de puissance, il est nécessaire de s'assurer que seul le fondamental est prépondérant dans ce fonctionnement non linéaire. La justification d'une telle approximation a été fournie expérimentalement par M. Goudelis [19]. Il a été démontré en effet que le taux de réjection fondamental sur harmonique, quel que soit l'harmonique considéré, reste égal au moins à $15 \mathrm{~dB}$ pour des taux de compression de l'ordre de $3 \mathrm{~dB}$ lorsque le transistor se trouve adapté au gain maximum en classe $A$.

iv) Le modèle représenté sur la figure 9 est décrit sous la forme d'un macro-composant non linéaire défini entre les électrodes de grille, de source et de drain. Il est associé au générateur d'impédance $Z_{\mathrm{s}}$ et à l'admittance $Y_{\mathrm{c}}$ calculées précédemment pour former un amplificateur dont le schéma est donné sur la figure 13. L'amplificateur est ainsi implanté dans le logiciel IMAG III. Les sources $E_{\mathrm{G}}$ et $E_{\mathrm{D}}$ constituent les polarisations continues. La tension $E_{\mathrm{G}}$ de grille est choisie dans notre étude pour imposer un courant statique de drain égal à $I_{\text {Dss }} / 2$. Le composant est alors dit fonctionner en classe A. La tension $E_{\mathrm{D}}$ de drain constituera un des paramètres d'étude. 
v) Compte tenu des notations de la figure 13 , on détermine ensuite la réponse du circuit à l'excitation sinusoïdale du générateur de grille. Lorsque la puissance délivrée par ce générateur sinusoïdal est telle que le TEC GaAs puisse travailler en régime de compression, nous déterminons par intégration numérique en régime temporel, les formes d'onde de tensions $v_{1}, v_{2}$, des courants $i_{1}, i_{2}$ et des produits $\left(v_{1}, i_{1}\right)$ et $\left(i_{2} \cdot v_{2}\right)$. Nous ne nous intéressons qu'au cas du régime permanent. Les variables courants et tensions sont périodiques et de forme non sinusoïdale, à cause du comportement non linéaire du transistor. Les paramètres de l'étude sont les valeurs des impédances de source et de charge, comme nous le préciserons dans le prochain paragraphe.

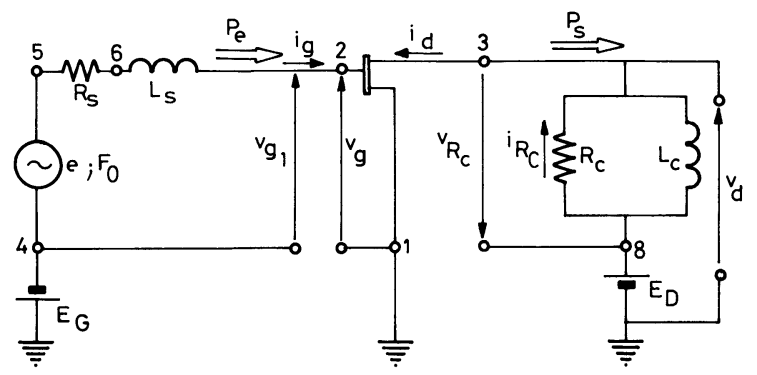

Fig. 13. - Schéma équivalent à constantes localisées, pour IMAG III, de l'étage amplificateur représenté sur la figure 11.

[Equivalent circuit, with lumped elements, used in the IMAG III numerical programme.]

Les puissances considérées sont : i) la puissance $P_{\mathrm{d}}$ disponible au générateur d'entrée, calculée par la relation (5), ii) la puissance $P_{\mathrm{e}}$ qui est absorbée dans la grille du TEC et iii) celle $P_{\mathrm{s}}$, qui est fournie à la charge $Z_{\mathrm{c}}$. Ces dernières sont respectivement égales aux valeurs moyennes des ondes de puissance, $V_{\mathrm{g}_{1}}(t)$. $i_{\mathrm{g}}(t)$ côté grille, et $V_{\mathrm{R}_{\mathrm{c}}}(t) . i_{\mathrm{R}_{\mathrm{c}}}(t)$ côté drain, calculées sur un nombre entier de périodes par les expressions (6) et (7).

Un exemple de forme d'onde de tension délivrée par l'étage amplificateur de la figure 13 , pour $2 \mathrm{~dB}$ de compression en puissance, est représenté sur la figure 14. Le comportement de cet amplificateur en régime temporel non linéaire est également traduit par les cycles dynamiques décrits par le courant de drain au cours du temps, en fonction de la tension drain-source (Fig. 15). Notons que l'apparition d'un courant de drain négatif est liée à l'existence de courants capacitifs dans certaines branches du modèle.

3. 3 SimULATION EN RÉGIME DE GRANDS SIGNAUX. 3.3.1 Principe de la méthode. - La méthode de simulation utilisée consiste, pour chaque valeur de la fréquence et de la tension d'alimentation de drain, à déterminer deux familles de courbes paramétrées par la valeur de puissance $P_{\mathrm{e}}$ :

1) La première famille concerne les variations de la puissance de sortie $P_{\mathrm{s}}$, en fonction de la partie

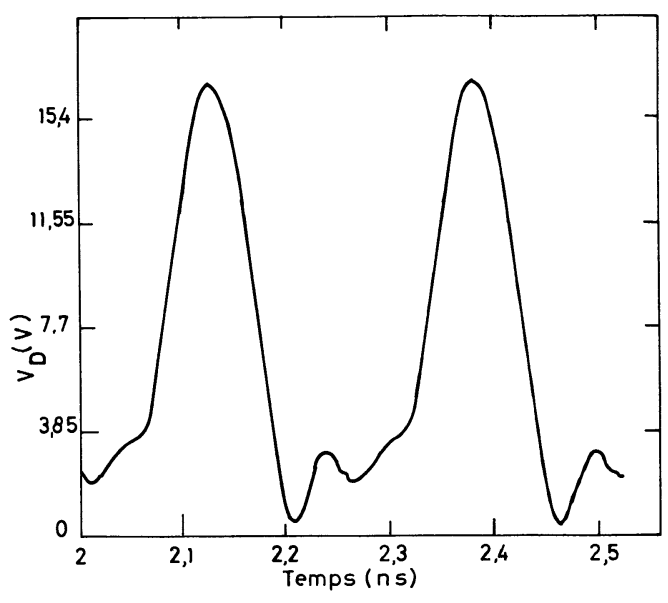

Fig. 14. - Forme d'onde théorique de la tension drain-source pour $2 \mathrm{~dB}$ de compression en puissance, THC $302(98), V_{\mathrm{D}}=7 \mathrm{~V}, V_{\mathrm{G}}=-2 \mathrm{~V}, P_{\mathrm{e}}=20 \mathrm{~mW}, F_{0}=$ $4 \mathrm{GHz}$.

[Drain-source voltage time dependence at $2 \mathrm{~dB}$ compression level.]

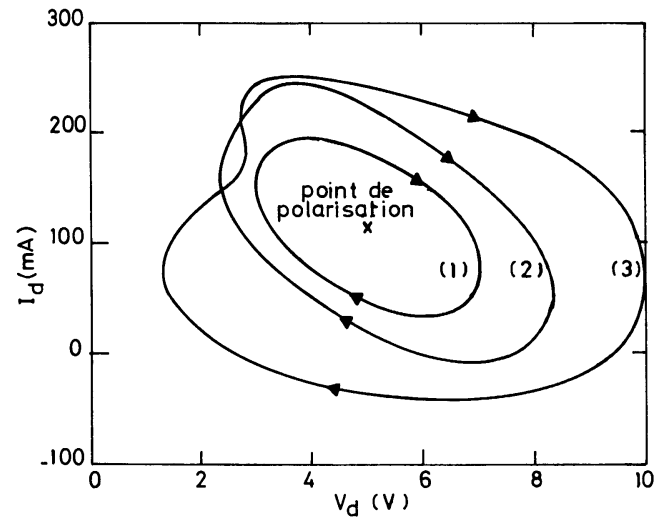

Fig. 15. - Cycles d'hystérisis décrits par le courant de drain en régime dynamique non linéaire. THC 302, $F_{0}=$ $6 \mathrm{GHz}, E_{\mathrm{D}}=5 \mathrm{~V}, V_{\mathrm{G}}=-2 \mathrm{~V}, I_{\mathrm{D}}=I_{\mathrm{DSs}} / 2$. (1) $P_{\mathrm{e}}=$ $5 \mathrm{~mW} ; P_{\mathrm{s}}=45 \mathrm{~mW}$ entrée adaptée, sortie adaptée, (2) $P_{\mathrm{e}}=20 \mathrm{~mW} ; P_{\mathrm{s}}=120 \mathrm{~mW}$ entrée adaptée, sortie adaptée, (3) $P_{\mathrm{e}}=20 \mathrm{~mW} ; P_{\mathrm{s}}=79 \mathrm{~mW}$ entrée adaptée, sortie désadaptée.

[The drain current hysteresis cycles on the output characteristics.]

réelle de l'admittance de charge $Y_{c}$. La partie imaginaire de $Y_{\mathrm{c}}$ est alors maintenue égale à sa valeur petits signaux définie dans le tableau II. Pour le transistor THC 302 (98), cette famille de courbes est représentée sur la figure 16 à $5 \mathrm{GHz}$. Il apparaît que ces courbes présentent toutes un maximum de puissance pour une valeur particulière de $R_{\mathrm{c}}$ notée $R_{\mathrm{c}_{0}}$;

ii) La deuxième famille trace les évolutions de la puissance de sortie en fonction de la partie imaginaire de l'admittance de charge $Y_{\mathrm{c}}$, ceci pour la même fréquence et les mêmes valeurs de la puis- 


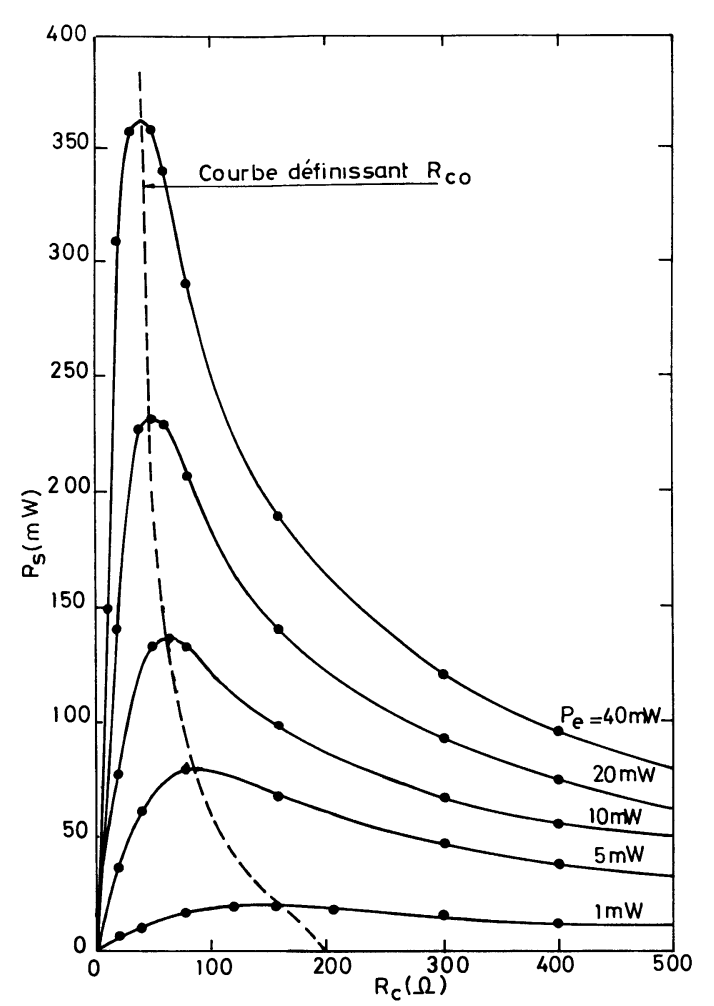

Fig. 16. - Caractéristiques théoriques de puissance de type $P_{\mathrm{s}}\left(R_{\mathrm{c}}\right), \mathrm{I}_{\mathrm{m}}\left(Y_{\mathrm{c}}\right)=$ Cte. Définition de la partie réelle, $1 / R_{\mathrm{c} 0}$, de l'admittance de charge grand signal. THC $302(98)$, $F_{0}=5 \mathrm{GHz}, V_{\mathrm{D}}=7 \mathrm{~V}, I_{\mathrm{D}}=120 \mathrm{~mA}, V_{\mathrm{G}}=-2 \mathrm{~V}$.

[Theoretical characteristics for the output power versus the load resistance.]

sance $P_{\mathrm{e}}$ et de la tension d'alimentation $E_{\mathrm{D}}$, que pour la première famille. La partie réelle de $Y_{c}$, alors prise comme second paramètre, a des valeurs qui correspondent aux divers maximums de la famille précédente. Un exemple de cette famille de courbes est représenté sur la figure 17. On note que ces courbes ont toutes un maximum se produisant pour la même valeur de $I_{m}\left(Y_{c}\right)$ qui est notée $I_{m}\left(Y_{c}\right)_{0}$.

$\mathrm{Au}$ cours de la simulation précédente, l'impédance de source $Z_{\mathrm{s}}$ est maintenue constante et égale à sa valeur adaptée en petits signaux. Dans cette condition d'adaptation, les diagrammes de la figure 18 qui comparent la puissance $P_{\mathrm{d}}$ disponible au générateur d'entrée (qui peut être modifiée en changeant la f.e.m. $e_{1}$ ) à la puissance $P_{\mathrm{e}}$, qui est effectivement absorbée par la grille, montrent que ces puissances diffèrent au maximum de $10 \%$. Par suite, on peut considérer que la condition d'adaptation reste vérifiée du côté entrée avec une tolérance meilleure que $10 \%$ pour une large gamme de valeurs de la résistance de charge $R_{\mathrm{c}}$. Par exemple, dans le cas du transistor THC 302, ces valeurs peuvent être comprises entre $20 \Omega$ et $400 \Omega$. Ce résultat théorique, en accord avec le comportement expérimental de l'impédance grand signal d'entrée $Z_{\text {in }}$ que nous avons reporté au para-

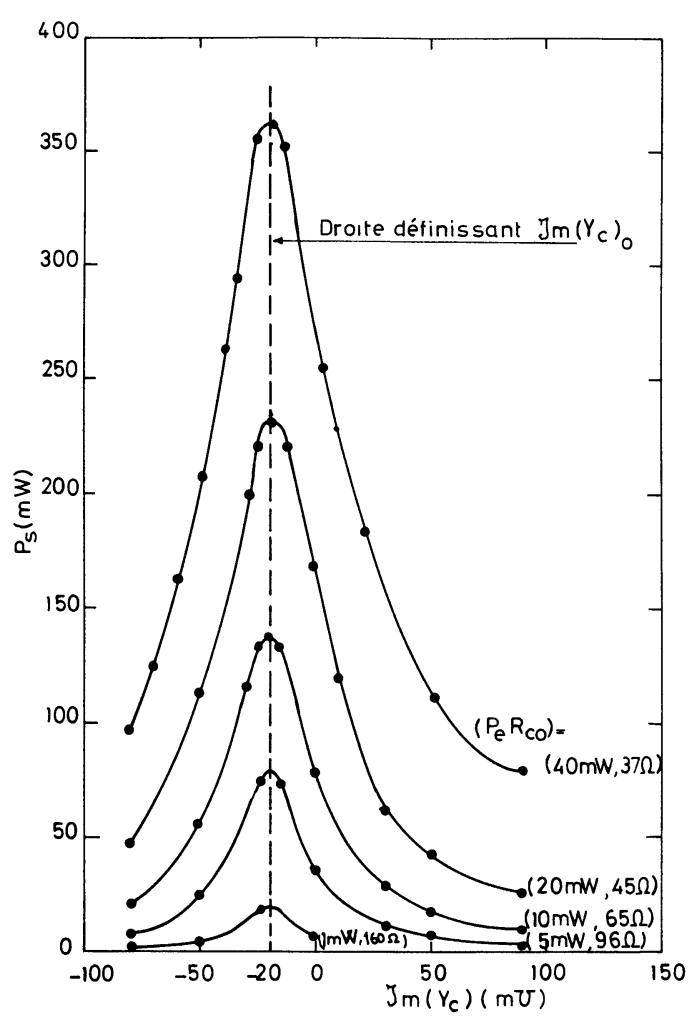

Fig. 17. - Variations théoriques de la puissance de sortie en fonction de la partie imaginaire de l'admittance de charge. Définition de la partie imaginaire de l'admittance de charge grand signal $\mathrm{I}_{\mathrm{m}}\left(Y_{\mathrm{c}}\right)_{0}$. THC 302(98), $F_{0}=5 \mathrm{GHz}, V_{\mathrm{D}}=7 \mathrm{~V}$, $I_{\mathrm{D}}=120 \mathrm{~mA}, V_{\mathrm{G}}=-2 \mathrm{~V}$.

[Theoretical variation of the output power as a function of the imaginary part of the load admittance. Definition of the large signal output admittance.]

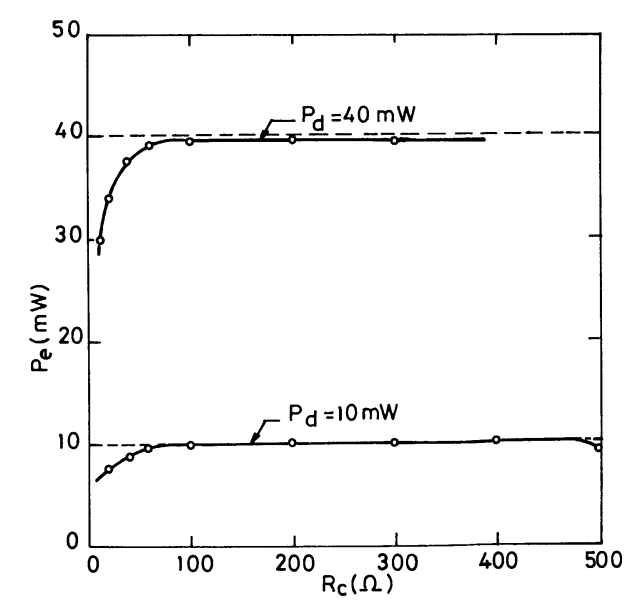

Fig. 18. - Variations théoriques de la puissance $P_{\mathrm{e}}$ qui entre dans le transistor en fonction de la résistance de charge $R_{\mathrm{c}}$. Le paramètre est la puissance disponible (au générateur d'entrée) $P_{\mathrm{d} \cdot}$ - — : valeurs calculées ; - - - : cas idéal avec adaptation parfaite ou $P_{\mathrm{e}}=P_{\mathrm{d}}$. THC 302 (18), $F_{0}=6 \mathrm{GHz}, V_{\mathrm{D}}=6 \mathrm{~V}, I_{\mathrm{D}}=I_{\mathrm{DSs}} / 2, V_{\mathrm{G}}=-2 \mathrm{~V}$.

[Theoretical variation of input power $P_{\mathrm{e}}$ as a function of the output load resistance $R_{\mathrm{c}}$. The power available from the input generator is considered as a parameter.] 
graphe 2.3.2, indique que l'obtention du maximum de puissance à la sortie du transistor ne dépend essentiellement que de l'impédance de charge.

3.3.2 Application à la détermination des impédances grands signaux du TEC. - 3.3.2.1 Influence de la valeur de puissance d'entrée. - Les impédances grands signaux sont définies lorsqu'il y a un transfert maximum de puissance vers la charge $Y_{c}(\S 2.2)$ avec un gain associé maximum. En confondant les puissances $P_{\mathrm{e}}$ et $P_{\mathrm{d}}$, cette condition d'adaptation est obtenue chaque fois qu'il existe un maximum sur les caractéristiques de puissance décrites sur les figures 16 et 17 précédentes :

- la partie réelle $\mathrm{R}_{\mathrm{e}}\left(Y_{\text {out }}\right)$ de l'admittance de charge grand signal $Y_{\text {out }}$ est égale à l'abscisse du maximum sur chaque courbe du type

$$
P_{\mathrm{s}}\left(R_{\mathrm{c}}\right) \mathrm{I}_{\mathrm{m}}\left(Y_{\mathrm{c}}\right)=\mathrm{Cte}
$$

de la figure 16 :

$$
\mathrm{R}_{\mathrm{e}}\left(Y_{\text {out }}\right)=\frac{1}{R_{\mathrm{c}_{\mathrm{o}}}}
$$

Un exemple des résultats obtenus sur le transistor THC 302 (98) à $5 \mathrm{GHz}$ est représenté sur la figure 19.

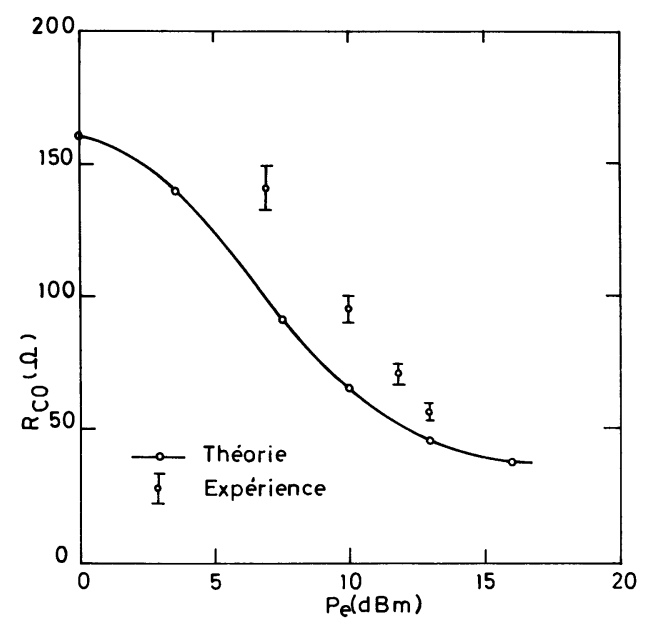

Fig. 19. - Variations théoriques et expérimentales de l'inverse de la partie réelle de l'admittance de charge grand signal. Mise en évidence de la saturation de celle-ci, THC $302(98), F_{0}=5 \mathrm{GHz}, V_{\mathrm{D}}=7 \mathrm{~V}, I_{\mathrm{D}}=I_{\mathrm{DSs}} / 2, V_{\mathrm{G}}=$ $-2 \mathrm{~V}$.

[Theoretical and experimental values of the real part of the large signal output admittance.]

On note, qu'en accord avec l'expérience, la quantité $\mathrm{R}_{\mathrm{e}}\left(Y_{\text {out }}\right)^{-1}$ décroît lorsque la puissance $P_{\mathrm{e}}$ augmente et tend vers une valeur constante pour les forts taux de compression;

- la partie imaginaire $I_{m}\left(Y_{\text {out }}\right)$ de l'admittance de sortie $Y_{\text {out }}$, qui est déterminée à partir de la figure 17, est indépendante du taux de compression en puissance et garde la valeur qu'elle a en régime d'adap- tation linéaire. Ce comportement de $\mathrm{I}_{\mathrm{m}}\left(Y_{\text {out }}\right)$ confirme le résultat expérimental décrit sur la figure 8 et signifie, finalement, que la puissance maximale que peut fournir un transistor à sa charge ne dépend essentiellement que de la partie réelle de l'admittance grand signal de sortie du TEC [14].

3.3.2.2 Prise en compte de l'influence des tensions continues de polarisation de grille et de drain. - La méthode décrite précédemment vient d'être appliquée lorsque les conditions de polarisation continues sont figées. Celle-ci peut encore être utilisée pour déterminer, d'une part le point de polarisation continue de grille $V_{G}$ qui permet d'obtenir la plus grande puissance sur le drain du transistor et, d'autre part pour définir l'influence de la tension continue de drain sur cette valeur de puissance.

i) Le choix du point de polarisation à $I_{\mathrm{DSs}} / 2$ : en désignant par $P_{\text {sopt }}$, la puissance maximale que peut fournir le composant lorsque les conditions d'adaptation sont réalisées, il apparaît que, en fonction de la tension grille source $V_{G}$ (Fig. 20), les variations

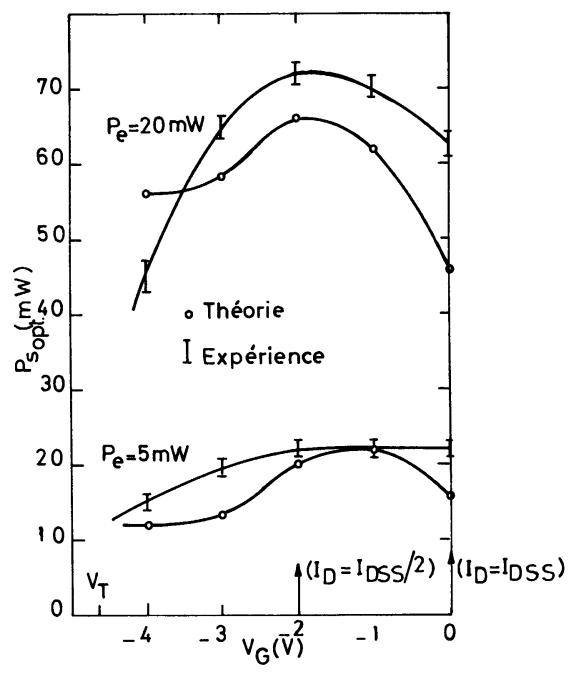

Fig. 20. - Variations théoriques et expérimentales de la puissance optimale dissipée dans la charge avec la tension continue grille-source. THC $302(18), F_{0}=8 \mathrm{GHz}, V_{\mathrm{D}}=$ $4 \mathrm{~V}$. Le paramètre est la puissance d'entrée $P_{\mathrm{e}}$.

[Maximum output power as a function of the DC gate source voltage.]

théoriques(obtenues par la méthodologie des figures 16 et 17) et expérimentales de $\boldsymbol{P}_{\text {sopt }}$ présentent un maximum pour une valeur de la tension de grille correspondant à un courant de drain égal à $I_{\mathrm{DSs}} / 2$. En fait, à faible niveau de puissance d'entrée $(5 \mathrm{~mW}$ pour le THC 302) cette courbe $P_{\text {sopt }}\left(V_{\mathrm{G}}\right)$ présente un maximum qui est très aplati, de sorte que le choix de la tension continue de polarisation de grille est peu critique; le transistor fonctionne en régime linéaire. Par contre, pour les valeurs élevées de la puissance d'entrée ( $20 \mathrm{~mW}$ pour le THC 302$)$ le maximum est très prononcé et montre la nécessité du choix judi- 
cieux de la polarisation pour le courant $I_{\mathrm{DSs}} / 2$. On notera que les différences observées entre les résultats théoriques et ceux de l'étude expérimentale sont de l'ordre de $20 \%$ au maximum;

ii) L'influence de la tension de drain sur la valeur de la puissance de sortie : la puissance transmise à la charge dépend de la f.e.m. du générateur continu du drain $E_{\mathrm{D}}$. Afin de voir comment elle en dépend, on reproduit les réseaux des figures 16 et 17 pour diverses valeurs de la f.e.m. $E_{\mathrm{D}}$. Le réseau de courbes de la figure 21 représente l'évolution de la puissance

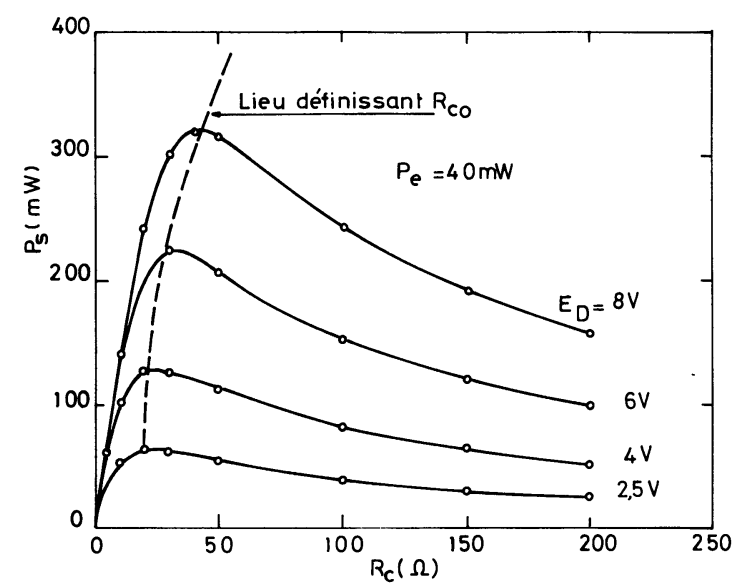

Fig. 21. - Variations théoriques de la puissance de sortie en fonction de la résistance de charge $R_{\mathrm{c}}$. Le paramètre est la tension d'alimentation continue de drain $E_{\mathrm{D}}$. THC 302(18), $F_{0}=6 \mathrm{GHz}, V_{\mathrm{G}}=-2 \mathrm{~V}$.

[Theoretical variations of the output power as a function of the load resistance. The DC drain voltage is considered as a parameter.]

de sortie, en fonction de la partie réelle de l'admittance de charge $R_{\mathrm{c}}$, lorsque la tension d'alimentation est un paramètre, la puissance $P_{\mathrm{e}}$ est figée et l'adaptation à l'entrée est effectuée. Les courbes $P_{\mathrm{s}}\left(R_{\mathrm{c}}\right)$ présentent toutes un maximum qui se produit pour une abscisse $R_{\mathrm{c}_{0}}$ dépendant du paramètre $E_{\mathrm{D}}$. Ces différents maxima définissent un lieu en pointillé sur la figure 21 qui, paramétré par la f.e.m. $E_{\mathrm{D}}$, fournit la puissance optimale $P_{\text {sopt }}$ qui peut être transmise à la charge.

La figure 22 représente les variations théoriques et expérimentales de la puissance $P_{\text {sopt }}$ en fonction de la tension continue d'alimentation $E_{\mathrm{D}}$ pour deux niveaux de puissance $P_{\mathrm{e}}$. Les courbes obtenues permettent de préciser que les variations de la puissance optimale fournie à la charge sont une fonction linéaire croissante de celles de la tension continue drain-source, dès que le canal est pincé. Rappelons toutefois, en tant que limitation pratique, que l'on n'est pas libre d'augmenter $E_{\mathrm{D}}$ au-delà de la valeur qui peut entraîner l'apparition du claquage par avalanche entre drain et grille ou entre drain et

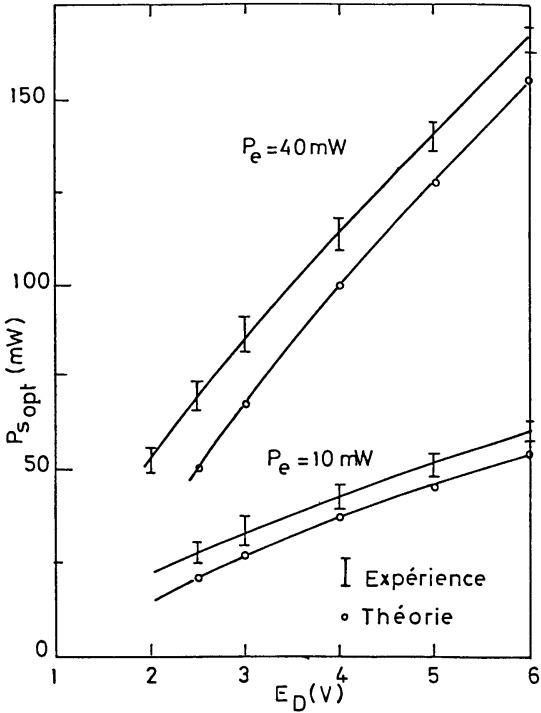

Fig. 22. - Variations théoriques et expérimentales de la puissance optimale délivrée à la charge avec la tension d'alimentation continue de drain. Le paramètre est la puissance d'entrée $P_{\mathrm{e}}$. THC $302(18), F_{0}=8 \mathrm{GHz}, V_{\mathrm{G}}=-2 \mathrm{~V}$.

[Experimental and theoretical output power as a function of the DC drain voltage. The input power is considered as a parameter.]

source lors du parcours du cycle d'hystérésis dynamique sur les caractéristiques de sortie.

3.3.2.3 Influence des divers paramètres. - L'abaque d'évolution de la puissance de sortie optimale, en fonction de la puissance d'entrée, de la tension d'alimentation de drain, de la partie réelle de l'admittance de charge, est obtenu en combinant les familles de courbes déterminées dans les trois paragraphes précédents. Un exemple des valeurs typiques pour le transistor THC 302 fonctionnant à la fréquence de $6 \mathrm{GHz}$ est tracé sur la figure 23 : l'axe horizontal

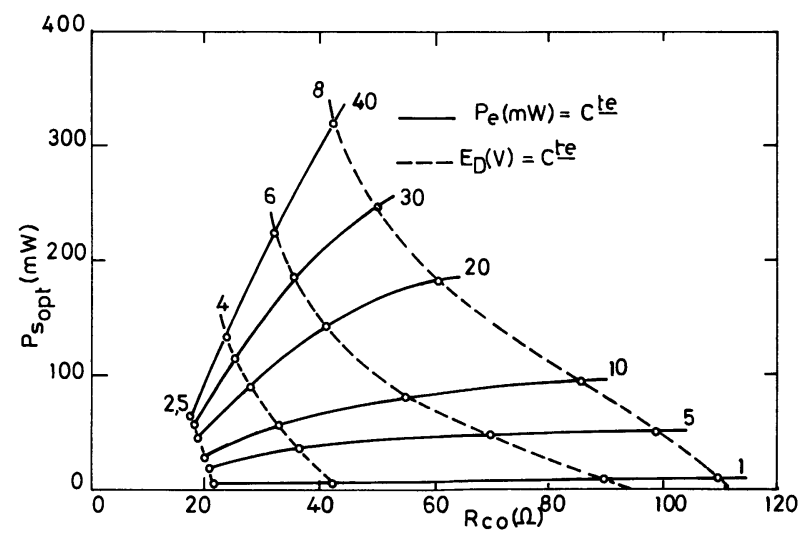

Fig. 23. - L'abaque de puissance optimale de sortie. Les paramètres sont : la puissance d'entrée et la tension continue d'alimentation de drain. THC 302 (18), $F_{0}=6 \mathrm{GHz}$, $I_{\mathrm{D}}=I_{\mathrm{DSs}} / 2, \quad V_{\mathrm{G}}=-2 \mathrm{~V}$.

[Maximum output power as a function of the input power and of the DC drain bias.] 
représente les valeurs de la partie réelle de la charge (en configuration parallèle), l'axe vertical donne la puissance maximale fournie par le transistor; les courbes en trait plein correspondent à un fonctionnement à puissance d'entrée constante, celles en pointillés sont déterminées pour chaque valeur de la tension d'alimentation de drain. Cet abaque résume les caractéristiques et fournit les limites de performances du transistor de puissance.

\subsubsection{Détermination des diagrammes d'impédances} à gain constant. - Ces diagrammes constituent la généralisation des études précédentes. Ils représentent, sur l'abaque de Smith, le lieu géométrique des impédances de charge du transistor qui, pour une fréquence et une puissance $P_{\mathrm{e}}$ données, correspond à des conditions de transfert de puissance telles que le gain en puissance et, par suite, la puissance de sortie restent constants. Ces divers lieux sont déterminés en construisant numériquement, suivant la même procédure de calcul des puissances et pour chaque valeur de la puissance d'entrée $P_{\mathrm{e}}$ (que l'on peut confondre avec $P_{\mathrm{d}}$ ), une surface dans l'espace à trois dimensions, $P_{\mathrm{s}}$ fonction de $R_{\mathrm{c}}$ et $\mathrm{I}_{\mathrm{m}}\left(Y_{\mathrm{c}}\right)$, schématisée sur la figure 24. En coupant cette surface

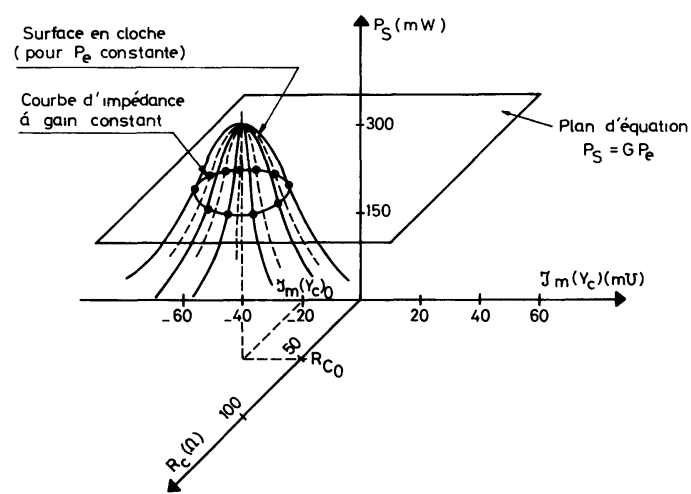

Fig. 24. - Détermination des diagrammes d'impédances à gain constant.

[Method for determining the constant power gain locus.]

par divers plans horizontaux correspondant chacun à une valeur particulière $\boldsymbol{P}_{\mathrm{s}} / \boldsymbol{P}_{\mathrm{e}}$, on obtient à l'intersection les courbes $R_{\mathrm{c}}-\mathrm{I}_{\mathrm{m}}\left(Y_{\mathrm{c}}\right)$, dites à gain constant. Ces caractéristiques transposées sous la forme d'impédance, en configuration série, sont ensuite reportées sur l'abaque de Smith. On pourra remarquer que les réseaux des figures 16 et 17 ne représentent, en fait, que l'intersection de ces diverses surfaces avec le plan vertical défini par la valeur particulière $\mathrm{I}_{\mathrm{m}}\left(Y_{\mathrm{c}}\right)_{0}$ prise par $I_{m}\left(Y_{c}\right)$.

En tant qu'exemple, les courbes de la figure 25 représentent les résultats obtenus sur le transistor de moyenne puissance THC 302 à la fréquence de $5 \mathrm{GHz}$ et pour une puissance $P_{\mathrm{e}}$ de $20 \mathrm{~mW}$. Ces lieux d'impédances de charge, ramenés sur l'abaque de Smith, présentent les formes suivantes : i) lorsque le

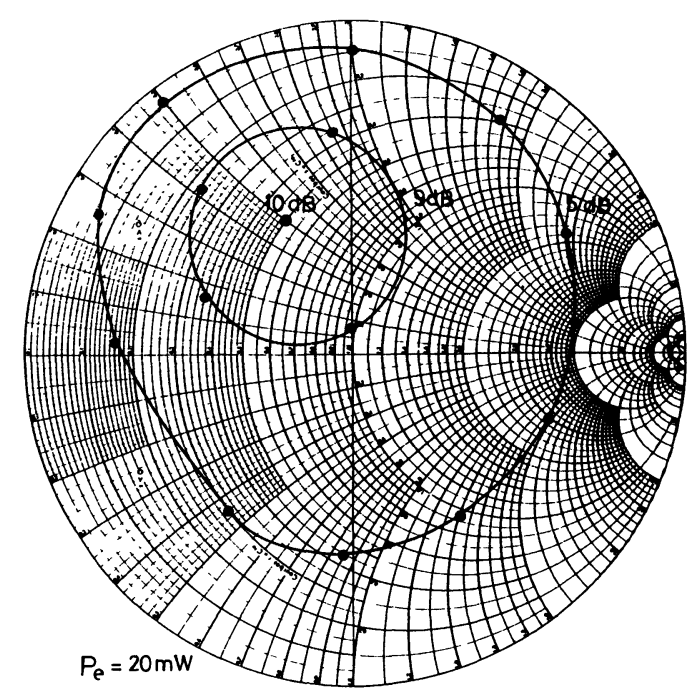

Fig. 25. - Diagrammes théoriques d'impédance de charge à gain constant. Le paramètre est le gain en puissance. Configuration série de l'impédance de charge, impédance caractéristique $50 \Omega . P_{\mathrm{e}}=20 \mathrm{~mW}$. THC $302(98), F_{0}=$ $5 \mathrm{GHz}$.

[The constant power gain locus plotted on the Simth chart.]

gain en puissance est maximum $\left(G=G_{\max }\right)$, c'est-àdire lorsque $P_{\mathrm{s}} / P_{\mathrm{e}}$ est maximum, le diagramme se réduit à un point dont la partie réelle et la partie imaginaire correspondent à la valeur de l'impédance de charge optimale en régime grand signal, ii) lorsque le gain est proche de sa valeur maximale $\left(G \approx G_{\mathrm{max}}\right)$, ces contours d'impédance ont une allure circulaire. Ce phénomène s'explique qualitativement, par le fait que les éléments non linéaires du modèle mathématique du transistor ne varient pas beaucoup et gardent sensiblement les valeurs qui imposent le gain maximum, et ceci quelle que soit la charge, iii) Lorsque le gain est très éloigné de sa valeur maximale $\left(G \ll G_{\max }\right)$, c'est-à-dire en régime de forte compression, le diagramme de charge devient une boucle ovale. Ce comportement traduit l'évolution des paramètres non linéaires du transistor, du régime linéaire au régime de saturation. Ces résultats théoriques de la figure 25 sont à mieux que $20 \%$ près, identiques à ceux relevés expérimentalement par R. Goudelis qui a tracé ces lieux par la méthode de la charge active [19].

3.3.4 Les caractéristiques $P_{\mathrm{s}}\left(P_{\mathrm{e}}\right)$. - Les caractéristiques traduisant la dépendance entre la puissance de sortie $P_{\mathrm{s}}$ et la puissance d'entrée $P_{\mathrm{e}}$ sont déduites des courbes $P_{\mathrm{s}}\left(R_{\mathrm{c}}\right) P_{\mathrm{e}}=$ Cte, précédemment obtenues (Fig. 16). Ce réseau de courbes permet de tracer les diverses caractéristiques théoriques $P_{\mathrm{s}}\left(P_{\mathrm{e}}\right)$ pour les conditions d'adaptation d'impédance suivantes : i) l'adaptation est réalisée pour des conditions de puissance d'entrée $P_{\mathrm{e}}$ figées; les courbes $P_{\mathrm{s}}\left(P_{\mathrm{e}}\right)$ théoriques sont obtenues en traçant une droite verticale passant par le maximum de la caractéristique $\boldsymbol{P}_{\mathrm{s}}\left(\boldsymbol{R}_{\mathrm{c}}\right)$ correspondant à la valeur de la puissance $\boldsymbol{P}_{\mathrm{e}}$ 
pour laquelle l'adaptation est réalisée. Aux points d'intersection, entre cette verticale et les diverses courbes, paramétrée en valeurs de $P_{\mathrm{e}}$, on lit les valeurs de $P_{\mathrm{e}}$ et de $P_{\mathrm{s}}$ pour obtenir la courbe $P_{\mathrm{s}}\left(P_{\mathrm{e}}\right)$. On traite ainsi le cas des adaptations fixes à faible ou à fort niveau de $P_{\mathrm{e}}$, ii) la caractéristique $P_{\mathrm{s}}\left(P_{\mathrm{e}}\right)$, lorsque l'adaptation est effectuée point par point est obtenue en relevant sur chaque courbe du réseau de la figure 16 , le maximum de la puissance de sortie en fonction de la puissance d'entrée correspondante. Un exemple de résultat obtenu (Fig. 26) présente, en accord avec l'expérience, une pente unité à faible niveau de $P_{\mathrm{e}}$ et traduit le mécanisme de compression en puissance avec une précision de l'ordre de $10 \%$.

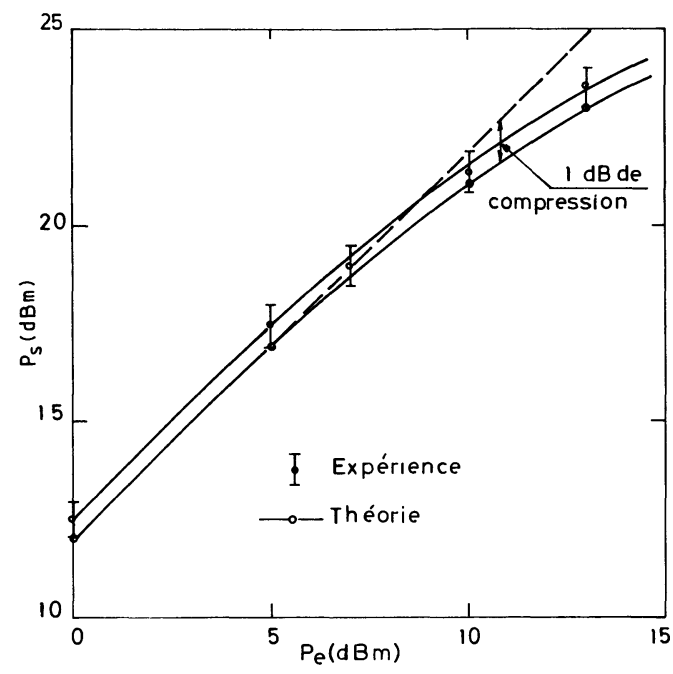

Fig. 26. - Caractéristiques $P_{\mathrm{s}}\left(P_{\mathrm{e}}\right)$ théoriques et expérimentales. Adaptation point par point. THC 302 (98), $F_{0}=5 \mathrm{GHz}, V_{\mathrm{D}}=7 \mathrm{~V}, I_{\mathrm{D}}=I_{\mathrm{DSs}} / 2, V_{\mathrm{G}}=-2 \mathrm{~V}$.

[Experimental and theoretical output power values as a function of the input power level. The matching conditions are realized for each value of the input power.]

4. Conclusion. - Cet article a été divisé en deux parties :

- au cours de la première partie, une méthode de caractérisation et d'identification du TEC GaAs en régime non linéaire a été développée. A partir du concept général de puissance, la notion d'impédances grands signaux d'un quadripôle évoluant en régime non linéaire a été introduite, puis associée aux caracté- ristiques de puissances permettant d'évaluer les paramètres qui sont à la base du calcul des amplificateurs hyperfréquences de puissance;

- La seconde partie a été consacrée à la description d'une méthodologie de simulation de ces amplificateurs à TEC GaAs, basée sur la détermination des diagrammes décrivant l'évolution de la puissance fournie par le transistor à sa charge, en fonction des parties réelle et imaginaire de celle-ci. L'approche théorique est effectuée dans le domaine temporel où les valeurs des diverses puissances sont calculées à partir des formes d'onde de courant et de tension.

Parmi les résultats obtenus, les points essentiels suivants peuvent être dégagés :

i) l'impédance d'entrée grand signal du transistor varie peu par rapport à celle de sortie, lorsque la puissance d'entrée $P_{\mathrm{e}}$ augmente ; elle reste voisine de sa valeur en régime petits signaux;

ii) quel que soit le niveau des puissances dans le transistor, la partie imaginaire de l'admittance grand signal de sortie reste quasi constante et égale à sa valeur en régime petits signaux;

iii) la partie réelle de cette admittance grand signal de sortie du TEC tend à prendre une valeur de saturation, lorsqu'on atteint les forts taux de compression en puissance;

iv) la puissance optimale délivrée à la charge n'est fonction principalement que de la partie réelle de l'admittance de charge, d'une part. D'autre part, elle est une fonction linéaire croissante de la tension d'alimentation continue de drain. Enfin, elle est maximale pour la condition de polarisation de grille, telle que le courant de drain soit égal à la valeur $I_{\text {Dss }} / 2$.

D'un point de vue pratique, la connaissance des impédances grands signaux permet de définir la configuration des circuits d'adaptation et est la base de conception de maquettes d'amplificateurs de puissance hyperfréquence.

Remerciements. - Les auteurs remercient leurs collègues du CRECO Microondes C.N.R.S. qui les ont aidés et qui ont participé à ce travail. Il s'agit de messieurs J. F. Sautereau du L.A.A.S.-C.N.R.S., G. Salmer du C.H.S. Lille, R. Soarès, B. Loriou, R. Périchon du C.N.E.T. Lannion, et J. L. Sédard du C.N.E.S.

\section{Bibliographie}

[1] Raucher, C. and Willing, H. A., Simulation of non linear microwave FET performance using a quasistatic model. IEEE Transaction on Microwave Theory and Techniques, Vol. MTT-27, oct. 1979, pp. 834-840.
[2] Yusuke Tajima, Beverly Wrona, Katsuhiko MishiMA, GaAs FET Large Signal model and its application to circuit designs. IEEE Transaction on Microwave Theory and Techniques. Vol. ED-28, fev. 1981, pp. 171-175. 
[3] Minasian, R. A., Large signal GaAs FET model and distorsion analysis. Electron. Lett. 14 no 6 (1978) 183-185.

[4] Azızı, C., Kamdem, J., Rossel, P., Modèles du transistor à effet de champ à l'Arséniure de Gallium utilisable dans le domaine temporel grand signal. Journées Nationales Microondes, Lille, juin 1979.

[5] KAMDEM, J., Le transistor à effet de champ à l'Arséniure de Gallium en régime non linéaire d'amplification hyperfréquence de puissance. Thèse 3e Cycle, Univ. Paul Sabatier, Toulouse (1981).

[6] Rossel, P., Azizi, C., Graffeuil, J., Modèles du transistor à effet de champ à l'Arséniure de Gallium. Rev. Polytech. 1372-4-78 (1977) 335-345.

[7] Azızı, C., Contribution à l'étude des propriétés statiques et dynamiques du transistor à effet de champ à grille Schottky. Thèse de 3e Cycle, Univ. Paul Sabatier, Toulouse (1976).

[8] Савот, J. J., Propriétés statiques du transistor à effet de champ à l'Arséniure de Gallium à grille Schottky. Thèse de 3e Cycle, Univ. Paul Sabatier, Toulouse (1974).

[9] Rossel, P., Tranduc, H., Graffeuil, J., Azizi, C., Phénomènes de relaxation dans les structures planes épitaxiales à l'Arséniure de Gallium. Rev. Phys. Appliquée 12 (1977) 1679-1694.

[10] GrafFeUIL, J., Le transistor à effet de champ à grille métallique sur Arséniure de Gallium. Propriétés statiques et dynamiques petits signaux. Analyse des phénomènes de bruit de fond. Thèse de Doctorat d'Etat, Univ. Paul Sabatier (1977).

[11] Azızı, C., Le transistor hyperfréquence à effet de champ à l'Arséniure de Gallium : modèles mathématiques pour la Conception Assistée par Ordinateur des circuits non linéaires. Thèse de Doctorat d'Etat, Univ. Paul Sabatier (1981).

[12] Willing, H. A., Raucher, C., De Santis, P., A technique for predicting large signal performance of a GaAs MESFET. IEEE Transaction on Microwave Theory and Techniques. MTT-26 (1978), pp. 10171023.
[13] Azızı, C., Rossel, P., Modèles mathématiques du transistor à effet de champ au GaAs pour la conception assistée par ordinateur des circuits. Acta Electron. 3 (1980) 23.

[14] Azizi, C., Kamdem, J., Graffeuil, J., Rossel, P., Computer simulations of non linear GaAs MESFET circuits. 6 Tth ESSCIRC 80, sept. 1980, Univ. Scientifique et Médicale Grenoble (France).

[15] Rossel, P., Azizi, C., Tranduc, H., Graffeuil, J., Sautereau, J. F., Modèles physiques pour la conception des fonctions logiques à l'Arséniure de Gallium. Rapport de contrat DGRST CCM 76-7802, no 1817 (1978).

[16] SoARes, R. A., Novel large signal S-Parameters measurement techniques aids GaAs MESFET power amplifier design. 7th Proc. European Microwave Conference, pp. 113-117 (1977).

[17] Shamsur, Mazumder, R. and van Der PuiJe, P. D., Two signal « method of measuring the large signal $S$-parameters of transistors. IEEE Transaction on Microwave Devices and Techniques. Vol. MTT 26 no 6 pp. 417-420 (June 1978).

[18] Reynaud, J. C., IMAG III : un système de simulation et d'optimisation à circuits électroniques. Thèse 3e Cycle, Institut National Polytechnique de Grenoble (1978).

[19] Goudelis, M., Caractérisation des transistors à effet de champ GaAs hyperfréquence de puissance en régime non linéaire. Thèse de Docteur-Ingénieur, Ecole Nationale Supérieure des Télécommunications (1981).

[20] TAKaYAMA, Y., A new load full characterisation method for microwave power transistors, in digest Techn. papers, IEEE MTT-S Int. Microwave Symp., 1976, p. $218-220$. 